\title{
Analytic surgery and analytic torsion
}

\author{
ANDREW HASSELL ${ }^{1}$
}

\begin{abstract}
Analytic surgery, as defined in [9] and [6], is a one-parameter metric deformation of a Riemannian manifold $M$, which stretches $M$ across a separating hypersurface $H$ in a cylindrical fashion; the singular limit is a complete manifold with asymptotically cylindrical ends, $\bar{M}$. In this paper, the analysis of [9] and [6] is used to study the behaviour of analytic torsion of unitary representations under analytic surgery. A gluing formula is obtained relating the analytic torsion of $M$ to the 'b-analytic torsion' ${ }^{b} T$ (a regularized analytic torsion on manifolds with boundary) of $\bar{M}$. This is then used to prove the Cheeger-Müller theorem, asserting the equality of analytic and Reidemeister torsion $\tau$ on closed manifolds, and to prove the following combinatorial formula for b-analytic torsion on odd dimensional manifolds with boundary:
\end{abstract}

$$
{ }^{b} T(N)=\tau(N) 2^{-1 / 4 \chi(\partial N)} .
$$

As a step in the proof, a Hodge-theoretic description of the MayerVietoris sequence for cohomology under analytic surgery is developed.

\section{Introduction.}

This paper follows on from the two earlier papers on analytic surgery [9] and [6], which will be referred to as 'Part I' and 'Part II' below. Analytic surgery is a method for studying surgery on manifolds as a limiting, rather than discrete, process. Let $M$ be a closed manifold, and $H \subset M$ a separating hypersurface with defining function $x$; that is, $H=\{x=0\}$ and $d x \neq 0$ on $H$. Let $h$ be a metric on $M$ and $\epsilon \in\left[0, \epsilon_{0}\right]$ a parameter. The family of metrics

$$
g_{\epsilon}=\frac{d x^{2}}{x^{2}+\epsilon^{2}}+h
$$

\footnotetext{
${ }^{1}$ This research was supported in part by a Sloan Doctoral Dissertation Fellowship
} 
is a smooth family for $\epsilon>0$ and has a singular limit at $\epsilon=0$, which is an 'exact b-metric' (see section 2) on $\bar{M}$, the disconnected manifold with boundary obtained by compactifying each component of $M \backslash H$ with a copy of $H . \bar{M}$ may be regarded as the result of 'cutting' $M$ at $H$, but it carries a complete metric, with the boundary geometrically at infinity. In Parts I and II, the spectral geometry of a family of generalized Laplacians associated with $g_{\epsilon}$ was analysed, and the analysis was applied to a gluing formula for the eta invariant for the Dirac operator on a spin manifold $M$. In the present paper, this analysis is applied to analytic torsion.

Analytic torsion $T$ is an invariant of a flat unitary bundle $E$ over a Riemannian manifold $M$ introduced by Ray and Singer in [18]. It is defined by formal analogy with a formula for Reidemeister torsion, or R-torsion, denoted $\tau$, a combinatorial invariant of a simplicial complex. Ray and Singer showed that $T(M, g)$ has the same formal properties as $\mathrm{R}$-torsion, the most important of which is that, in a suitable sense, it is independent of the metric, and is thus a manifold invariant. They conjectured that these two torsions are equal. This was proved several years later by Cheeger and Müller independently in [5] and [15]. In recent years, several more proofs and generalizations of this result have appeared. Vishik [20], [21] has established the relationship between analytic torsion, defined using classical boundary conditions, and R-torsion on manifolds with corners. Burghelea, Friedlander and Kappeler [3] obtained a new proof using Witten's deformation of the de Rham complex via a Morse function. Müller in [16] extended the result to flat bundles $E$ assuming only that the determinant bundle $\operatorname{det} E$ is unitary. Bismut and Zhang [1] proved a further generalization when the bundle $E$ is not necessarily unitary; $\log (T / \tau)$ is then given by the integral of a local 'anomaly'. The formula (2) for b-analytic torsion fits into the scheme conjectured by Lück in [8], with a boundary contribution to the logarithm of torsion given by $\log 2 / 2$ times the index of an induced operator on the boundary. However the sign of this boundary contribution appears to be reversed relative to Lück's and Vishik's; see Example 8. (For the purpose of comparison, note that Lück's normalization of torsion is the square of that used here.)

In another direction, Klimek and Wojciechowski in [7] have studied the 'adiabatic limit' of analytic torsion on manifolds with boundary (that is, the limit when a cylinder of length $R \rightarrow \infty$ is attached to the boundary) in the case when the boundary Laplacian is invertible.

Since analytic torsion and Reidemeister torsion are both differential invariants, most proofs of their equality proceed by establishing that they have the same gluing formula under surgery, and then using this to compare the 
two torsions for an arbitrary manifold to one for which the result is known. In the present paper, this program is carried out for analytic surgery. But since the manifold with boundary $\bar{M}$ resulting from the 'cut' in analytic surgery carries a complete metric, the Laplacian on $\bar{M}$ has continuous spectrum; this means that the heat kernel on $\bar{M}$ is not trace class, so the analytic torsion on $\bar{M}$ cannot be defined by the usual formula (8) involving the trace of the heat kernel. However, a regularized analytic torsion, 'b-analytic torsion', was defined in [11] using the 'b-Trace' to regularize the trace of the heat kernel (see section 3). The gluing formula (30) expresses $T(M, E)$ in terms of ${ }^{b} T(\bar{M}, E)$ and other geometric data at $\epsilon=0$. By comparing to a standard surgery formula for R-torsion, the following formula is obtained for their ratio:

Theorem 1. If $M$ is odd dimensional, the difference $\log T-\log \tau$ obeys the surgery formula

$$
\log \frac{T\left(M, E, g_{\epsilon}\right)}{\tau\left(M, E, g_{\epsilon}\right)}=\log \frac{{ }^{b} T\left(\bar{M}, E, g_{0}\right)}{\tau\left(\bar{M}, E, g_{0}\right)}+\frac{1}{2} \chi_{E}(H) \log 2,
$$

where $\chi_{E}(H) \equiv \chi(H) \cdot \operatorname{rank} E$ is the Euler characteristic of the cohomology of $H$ with coefficients in $E$.

From this the Cheeger-Müller theorem follows using Cheeger's approach (also mentioned in [15]), transforming two copies of any manifold $M$ to $S^{n}$ by a series of Morse surgeries. Given this result the following combinatorial formula for b-analytic torsion is almost immediate:

Theorem 2. For an odd dimensional manifold with boundary $N$, with flat unitary bundle $E$ and exact b-metric $g$,

$$
{ }^{b} T(N, E, g)=2^{-\chi_{E}(\partial N) / 4} \tau(N, E, g) .
$$

This paper is organized as follows. In section 2 the results in Parts I and II which are relevant to this paper are discussed. In section 3 analytic, banalytic and R-torsion are discussed in detail. In section 4 a Hodge version of the Mayer-Vietoris sequence for analytic surgery is developed; this is an important requisite for deriving the surgery formulae, as well, perhaps, as being of independent interest. In sections 5 and 6 the gluing formulae for analytic and R-torsion, respectively, are derived, and by comparing these Theorem 1 is obtained. Finally, in section 7 Theorem 1 is applied to give another proof of the Cheeger-Müller theorem and to derive the combinatorial formula (2) for b-analytic torsion. 


\section{Analytic Surgery.}

Let $N$ be a manifold with boundary, and $x$ a boundary defining function. An exact b-metric on $N$ is a metric of the form

$$
\frac{d x^{2}}{x^{2}}+h
$$

where $h$ is a smooth metric. In terms of $t=\log x$ the metric looks like $d t^{2}+h$, so this form of metric gives $N$ the geometric structure of a manifold with asymptotically cylindrical ends, with $\log x \rightarrow-\infty$ approximately arc length along the end. In analytic surgery, the family of metrics (1) degenerates to an exact b-metric on $\bar{M}$. The geometric picture is that as $\epsilon \rightarrow 0$, a long cylindrical neck develops across $H$ with length $2 \sinh ^{-1}(1 / \epsilon)+O(1) \rightarrow \infty$. The study of analytic surgery was initiated in [9] (Part I) and continued in [6] (Part II), motivated by the study of a similar family of metrics in [10] (degenerating to an incomplete conic metric). The main objects of study were the resolvent and heat kernel of a a family of generalized Laplacians associated to $g_{\epsilon}$. Here, the results of those papers are applied to analytic torsion.

As the analytic torsion and b-analytic torsion are defined in terms of the heat kernel by (8) and (12), (13), determining the behaviour of analytic torsion involves understanding the behaviour of the heat kernel $e^{-t \Delta_{\epsilon}}$ as $\epsilon \rightarrow 0$. In Parts I and II this was done using different methods for short time $(t \leq C)$ and long time $(t \geq C)$ behaviour. The short time behaviour was treated in Part I via the asymptotic expansion of the heat kernel near the diagonal as $t \downarrow 0$. The long time behaviour was treated by constructing the resolvent $\left(\Delta_{\epsilon}-\lambda^{2}\right)^{-1}$ and using the functional calculus:

$$
e^{-t \Delta_{\epsilon}}=\frac{i}{2 \pi} \int_{\gamma} e^{-t \lambda^{2}}\left(\Delta_{\epsilon}-\lambda^{2}\right)^{-1} 2 \lambda d \lambda,
$$

where $\gamma$ is a contour enclosing the spectrum.

The analysis of Part I was carried out under the assumption that the Laplacian $\Delta_{H}$ on $H$ is invertible, that is, that the first eigenvalue $\sigma_{0}$ of $\Delta_{H}$ is positive. This assumption makes no essential difference to the short time behaviour, but greatly simplifies the long time behaviour of the heat kernel, which was indeed the reason for making that assumption. For if $\sigma_{0}>0$ then zero is below the continuous spectrum of the Laplacian on $\bar{M}$, which is the interval $\left[\sigma_{0}, \infty\right)$. This means that the spectrum of $\Delta_{\epsilon}$ remains discrete near zero as $\epsilon \rightarrow 0$, and so the heat kernel is exponentially decreasing as $t \rightarrow \infty$ 
(up to finite rank) uniformly in $\epsilon$. When $\Delta_{H}$ has null space, that is $\sigma_{0}=0$, the situation is quite different. The heat kernel $e^{-t \Delta_{\epsilon}}$ then has an expansion in powers of $t^{-j / 2}$, for $j \geq 0$, as $t \rightarrow \infty$ ([11, chapter 7] or [Part II, section 8]). This makes the limit of analytic torsion a more delicate matter, and indeed there is an 'extra' term in the surgery formula for analytic torsion from the small eigenvalues, those going to zero with $\epsilon$.

\subsection{The resolvent under surgery.}

As it is the small eigenvalues that dominate the behaviour of the heat kernel as $t \rightarrow \infty$, to obtain the long time behaviour of the heat kernel from the integral (3) it is necessary to understand the resolvent $\left(\Delta_{\epsilon}-\lambda^{2}\right)^{-1}$ as $\lambda$ and $\epsilon$ both approach zero. In Parts I and II this was done using the approach outlined in [13], in which the Schwartz kernel of the inverse of an elliptic operator is constructed on a blown-up version of its natural domain to resolve singularities in the operator. In this situation the operator $\Delta_{\epsilon}$ is degenerate at $x=0, \epsilon=0$; the vector field $\sqrt{x^{2}+\epsilon^{2}} \partial_{x}$ which is associated with $\Delta_{\epsilon}$ is singular there. In Part I, the single surgery space $X_{s}$ was defined by

$$
X_{s}=\left[M \times\left[0, \epsilon_{0}\right] ; H \times\{0\}\right]
$$

The notation means that the submanifolds listed after the semicolon are to be blown up in the given order. The vector field $\sqrt{x^{2}+\epsilon^{2}} \partial_{x}$ lifted to $X_{s}$ is smooth, and the two components $M_{ \pm}$of $\bar{M}$ are separated in $X_{s}$. In fact the boundary of $X_{s}$ consists of two parts, $B_{0}=\bar{M}$, which is the disjoint union of $M_{+}$and $M_{-}$, and the face $B_{1}$, a compactified copy of $H \times \mathbb{R}$, coming from the blowup. The resolvent $\left(\Delta_{\epsilon}-\lambda^{2}\right)^{-1}$ is constructed on the surgery double space $X_{s}^{2}$, a double-space analogue of $X_{s}$ (that is, with two copies of $M$ ):

$$
X_{s}^{2}=\left[M^{2} \times\left[0, \epsilon_{0}\right] ; H \times H \times\{0\} ; H \times M \times\{0\} ; M \times H \times\{0\}\right]
$$

for $\lambda$ away from the continuous spectrum $\left[\sigma_{0}, \infty\right)$ of $\Delta_{0}$.

In Part II, the assumption of invertibility of $\Delta_{H}$ is removed, and then further blowups are necessary to construct the resolvent as $\lambda$ and $\epsilon$ both approach zero (which is now the bottom of the continuous spectrum). To see why further blowups are necessary, consider the following example.

Example 3. Consider the resolvent $\left(\Delta-\lambda^{2}\right)^{-1}$ on a circle of length $L$. Let $r$ be an arclength coordinate. Then the kernel of the resolvent (regarded as 
a half density) is

$$
\left(\Delta-\lambda^{2}\right)^{-1}=\left(\frac{\sin \lambda\left|r-r^{\prime}\right|}{2 \lambda}+\frac{\cos \lambda\left(r-r^{\prime}\right)}{2 \lambda \tan \lambda L}\right)\left|d r d r^{\prime}\right|^{\frac{1}{2}}, \quad-L \leq r-r^{\prime} \leq L .
$$

To connect this with surgery, assume that $L$ is a function of $\epsilon$ : $L=$ $2 \sinh ^{-1}(1 / \epsilon)$, the approximate growth rate of the length of the cylinder in analytic surgery. Thus the variable $\sinh ^{-1}(1 / \epsilon)$ is a more geometric parameter than $\epsilon$ itself; for convenience define ias $\epsilon=1 / \sinh ^{-1}(1 / \epsilon)$ ('inverse arc-sinh'), which goes to zero with $\epsilon$, but only logarithmically. For $\lambda$ away from the continuous spectrum $[0, \infty)$ one may replace $\tan \lambda L$ by its limiting value $i \operatorname{sgn} \operatorname{Im} \lambda$ as $L \rightarrow \infty$. However, if $\lambda$ and $\epsilon$ are both allowed to approach zero, then the limit is a function of the limiting ratio $\lambda /$ ias $\epsilon$. Thus it is necessary to introduce the scaled spectral parameter $z=\lambda /$ ias $\epsilon$. Having done that, one should also introduce a rescaled arclength variable $s=r$ (ias $\epsilon)$, and then the resolvent may be written

$$
\left(\Delta-\lambda^{2}\right)^{-1}=\frac{1}{\operatorname{ias} \epsilon}\left(\frac{\sin z\left|s-s^{\prime}\right|}{2 z}+\frac{\cos z\left(s-s^{\prime}\right)}{2 z \tan z}\right)\left|d \mathrm{vol} d \mathrm{vol}^{\prime}\right|^{\frac{1}{2}} .
$$

This is now meromorphic in $z$ and (ias $\epsilon)^{-1}$ times a smooth (conormal) density down to ias $\epsilon=0$.

It is then reasonable to expect that the resolvent under surgery will be a nice (conormal) function of rescaled arclength along the cylinder, $s=$ (ias $\epsilon) \sinh ^{-1}(x / \epsilon)$ and $s^{\prime}$, as $\lambda$ and $\epsilon$ approach zero. Now it is easy to explain why further blowups on $X_{s}$ are necessary: $s$ is not a smooth function - in fact, not even continuous - on $X_{s}$. It takes the value \pm 1 on $M_{ \pm}$and the value 0 on $B_{1}$.

The logarithmic single surgery space $X_{\mathrm{Ls}}$ of Part II is a blown-up version of $X_{s}$ on which $s$ is a smooth function. It is defined by

$$
X_{\mathrm{Ls}}=\left(\left(X_{s}\right)_{\log }\right)_{\mathrm{tb}} .
$$

The subscript log means, by definition, that all boundary defining functions $\rho$ are replaced by ilg $\rho \equiv 1 /(\log 1 / \rho)$; the subscript tb indicates that all boundary hypersurfaces of codimension at least two (here just the intersection $B_{0} \cap B_{1}$ ) are to be blown up. In this case, one new face $B_{2} \cong H \times[-1,0]_{s} \cup H \times[0,1]_{s}$ is created (note that ilg $\rho$ and ias $\rho$ are smooth functions of each other and equal to first order at $\rho=0$ ). It turns out that the combined operations of $\log$ and tb have good functorial properties; see [6, section 2]. The resolvent with scaled spectral parameter, 
$\left(\Delta_{\epsilon}-(\operatorname{ias} \epsilon)^{2} z^{2}\right)^{-1}$, is constructed on the double space analogue of $X_{\mathrm{Ls}}$, $X_{\mathrm{Ls}}^{2}$ :

$$
X_{\mathrm{Ls}}^{2}=\left(\left(X_{s}^{2}\right)_{\log }\right)_{\mathrm{tb}}
$$

in section 6 of Part II.

\subsection{Reduced normal operator.}

Corresponding to the new face $B_{2}$ is a new model operator on that face. In fact the model operator of $\Delta_{\epsilon}$ occurs in two different places in Taylor series in ias $\epsilon$ off $B_{2}$ : near $B_{2}, \Delta_{\epsilon} \sim \Delta_{H}+(\operatorname{ias} \epsilon)^{2} D_{s}^{2}+O\left((\operatorname{ias} \epsilon)^{\infty}\right)$, where $s \in[-1,1]$ is the coordinate above. Hence

$$
\Delta_{\epsilon}-(\operatorname{ias} \epsilon)^{2} z^{2} \sim \Delta_{H}+(\operatorname{ias} \epsilon)^{2}\left(D_{s}^{2}-z^{2}\right) .
$$

On functions on $B_{2}$ valued in $C^{\infty}\left([-1,1]_{s} ;\right.$ null $\left.\Delta_{H}\right),\left(D_{s}^{2}-z^{2}\right)$ is the leading part. The operator $D_{s}^{2}$ comes with natural boundary conditions at $s= \pm 1$ which makes it into a self-adjoint operator on null $\Delta_{H}$-valued functions on $[-1,1]_{s}$, the reduced normal operator, denoted $\mathrm{RN}(\Delta)$. The boundary conditions are of mixed Dirichlet-Neumann type. The reduced normal operator is of prime importance for the calculations in this paper.

To describe the reduced normal operator, first recall some facts about the extended $L^{2}$ null space of $\Delta_{M_{ \pm}}$(with respect to the measure $d g_{0}$ ). The extended $L^{2}$ null space consists of the sections $v$ such that $\Delta_{M_{ \pm}} v=0$ and $v \in x^{-\delta} L^{2}\left(M_{ \pm}\right)$for all $\delta>0$. Since the measure $d g_{0}$ behaves as $d x / x . d y$ as $x \rightarrow 0$, functions which are bounded and nonvanishing at $x=0$ are not in $L^{2}$, but are in extended $L^{2}$. Near the boundary, $\Delta_{M_{ \pm}} \sim-\left(x \nabla_{x}\right)^{2}+\Delta_{H}$. It is shown in [11, chapter 5] that such $v$ have an expansion near $x=0$ :

$$
v \sim(\log x) v_{1}(y)+v_{0}(y)+v^{\prime}, \quad v^{\prime} \in L^{2}, v_{i} \in \operatorname{null}\left(\Delta_{H}\right) .
$$

Moreover, defining subspaces of $\operatorname{null}\left(\Delta_{H}\right)$

$$
\begin{gathered}
\Lambda_{ \pm}^{N}=\left\{v_{1} \mid \exists v \sim(\log x) v_{1}(y)+v_{0}(y)+v^{\prime}, \Delta v=0, v^{\prime} \in L^{2}\right\} \\
\Lambda_{ \pm}^{D}=\left\{v_{0} \mid \exists v \sim v_{0}(y)+v^{\prime}, \Delta v=0, v^{\prime} \in L^{2}\right\}
\end{gathered}
$$

then $\Lambda_{ \pm}^{N}$ and $\Lambda_{ \pm}^{D}$ are orthocomplements in null $\left(\Delta_{H}\right)$ for each choice of sign. They are the leading coefficients of logarithmically growing and bounded extended $L^{2}$ null space, respectively. They are determined by the value of the scattering matrices $S_{ \pm}(\lambda)$ (see [11, chapter 6] or [Part II, section 6]) at 
$\lambda=0$. In fact, for $\lambda$ small, $S_{ \pm}(\lambda)$ is an $\operatorname{End}\left(\operatorname{null}\left(\Delta_{H}\right)\right)$-valued function of $\lambda$ and

$$
S_{ \pm}(0)=\operatorname{proj} \Lambda_{ \pm}^{D}-\operatorname{proj} \Lambda_{ \pm}^{N}
$$

In Part II, section 3, it is shown that if $u \in C^{\infty}\left(X_{\mathrm{Ls}}\right)$ is an 'approximate surgery eigenfunction', that is,

$$
\left(\Delta_{\epsilon}-(\operatorname{ias} \epsilon)^{2} z^{2}\right) u=O\left((\operatorname{ias} \epsilon)^{3}\right),
$$

then $\bar{u}=u \uparrow B_{2}$ satisfies the equation

$$
\begin{gathered}
\left(D_{s}^{2}-z^{2}\right) \bar{u}=0, \\
\text { and }\left.\bar{u}\right|_{s=-1} \in \Lambda_{-}^{D},\left.D_{s} \bar{u}\right|_{s=-1} \in \Lambda_{-}^{N}, \\
\left.\bar{u}\right|_{s=+1} \in \Lambda_{+}^{D},\left.D_{s} \bar{u}\right|_{s=+1} \in \Lambda_{+}^{N} .
\end{gathered}
$$

This comes from analysing the Taylor series of $u$ at the boundary of $X_{\mathrm{Ls}}$. The reduced normal operator $\mathrm{RN}(\Delta)$ is the operator $D_{s}^{2}$ with the boundary conditions (6). In Part II, section 6, it is shown that

Proposition 4. The small eigenvalues $\lambda(\epsilon)$ have the behaviour $\lambda(\epsilon)=$ $(\operatorname{ias} \epsilon)^{2} z^{2}(\epsilon)+o\left((\operatorname{ias} \epsilon)^{2}\right)$, where $\lim _{\epsilon \rightarrow 0} z^{2}(\epsilon)$ is equal to either 0 or an eigenvalue of $\mathrm{RN}(\Delta)$ or infinity.

\subsection{Heat kernel under surgery.}

In Parts I and II the heat kernel for small times and the heat kernel for long times were constructed in different ways. The heat kernel for small times was constructed in Part I by the usual Hadamard parametrix method adapted to surgery geometry, and works in the general case (with no assumption on invertibility of $\Delta_{H}$ ). The heat kernel on the diagonal for small times $(t \leq 1)$ lives on the space $X_{s} \times[0,1]_{\tau}$ (where $\tau=\sqrt{t}$ ). To make it easier to compare with the heat kernel for large times, it is a good idea to lift it to $\Delta_{\mathrm{LHs}}^{[0,1]}=X_{\mathrm{Ls}} \times[0,1]_{\tau}$. For large times $(t \geq 1)$, the heat kernel was constructed in Part II via the contour integral (3), and lives on the more complicated space

$$
\Delta_{\mathrm{LHs}}^{[1, \infty]}=\left[X_{\mathrm{Ls}} \times[1, \infty]_{\tau} ; B_{2} \times\{\tau=\infty\} ; B_{0} \times\{\tau=\infty\} ; B_{1} \times\{\tau=\infty\}\right] .
$$

The notation $[1, \infty]_{\tau}$ denotes the compactification of $[1, \infty)_{\tau}$ at infinity with boundary defining function $1 / \tau$ at infinity. The full heat kernel (on the 
diagonal) then lives on the space $\Delta_{\mathrm{LHs}}$ which is the union of $\Delta_{\mathrm{LHs}}^{[0,1]}$ and $\Delta_{\text {LHs }}^{[1, \infty]}$ along $\tau=1$. The blowups create extra faces at infinite time, on which the heat kernel has transitional behaviour between the polynomial decay of the heat kernel on $\bar{M}$ and the rapid decay on $M$ (see figure).

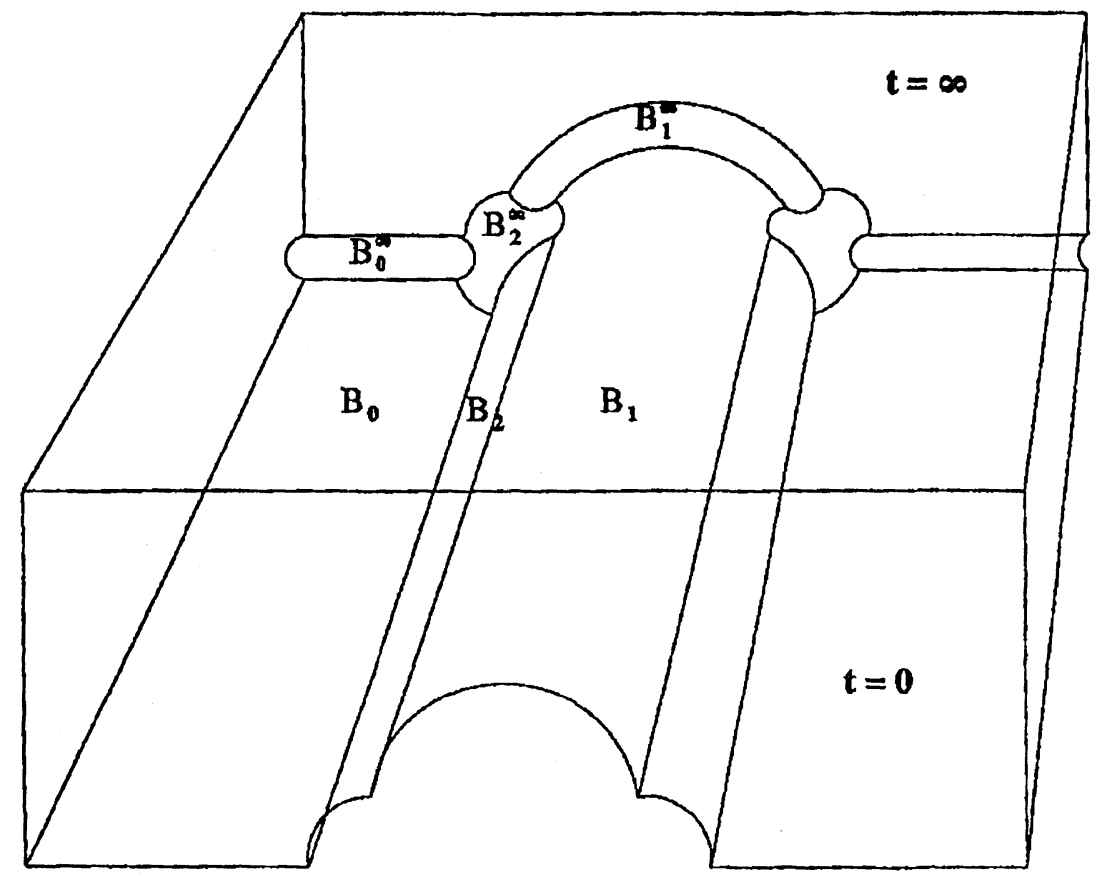

Denote by $B_{0}, B_{1}$ and $B_{2}$ the three boundaries at $\epsilon=0$ lifted from $X_{\mathrm{Ls}} \times[0, \infty]_{\tau}$, denote by $B_{0}^{\infty}, B_{1}^{\infty}, B_{2}^{\infty}$ the three new boundaries at $t=\infty$ created by blowup and denote by $B_{t \infty}$ the lift of $X_{\mathrm{Ls}} \times\{\tau=\infty\}$. Let $\rho_{0}, \rho_{1}$, $\rho_{2}, \rho_{0}^{\infty}, \rho_{1}^{\infty}, \rho_{2}^{\infty}$ be corresponding boundary defining functions and $\rho_{\mathrm{tf}}$ be a boundary defining function for the boundary at $\tau=0$. After multiplying by the formal density factor $\left|d(\operatorname{ias} \epsilon)(\operatorname{ias} \epsilon)^{-2} d t / t\right|$, the heat kernel restricted to the diagonal is in $\rho_{\mathrm{tf}}^{-n} C^{\infty}\left(\Delta_{\mathrm{LHs}} ; \Omega_{D}\right)$, where the $D$-density bundle is defined by $\Omega_{D}\left(\Delta_{\mathrm{LHs}}\right)=\left(\rho_{0} \rho_{1} \rho_{0}^{\infty} \rho_{1}^{\infty}\right)^{-1}\left(\rho_{2} \rho_{2}^{\infty}\right)^{-2} \Omega_{b}\left(\Delta_{\mathrm{LHs}}\right)$. The heat kernel has the following behaviour at $B_{0}, B_{1}, B_{2}$ and $B_{2}^{\infty}$ :

At $B_{0}, e^{-t \Delta_{\epsilon}}$ restricts to $e^{-t \Delta_{\bar{M}}}+O($ ias $\epsilon)$

At $B_{1}, e^{-t \Delta_{\epsilon}}$ restricts to $e^{-t \Delta_{\bar{H}}}+O($ ias $\epsilon)$

At $B_{2}, e^{-t \Delta_{\epsilon}}$ restricts to $e^{-t \Delta_{\bar{H}}}+O($ ias $\epsilon)$

At $B_{2}^{\infty}, e^{-t \Delta_{\epsilon}}$ is $O($ ias $\epsilon)$,

$(\text { ias } \epsilon)^{-1} e^{-t \Delta_{\epsilon}}$ restricts to $e^{-T \mathrm{RN}(\Delta)}(s, s)$. 
The notation in the fourth line has the following meaning. First, $T=$ $t(\text { ias } \epsilon)^{2}$ is a rescaled time variable along the boundaries at $t=\infty$, ias $\epsilon=0$. Second, the heat kernel $e^{-T \mathrm{RN}(\Delta)}$ of the reduced normal operator is an End(null $\Delta_{H}$ )-valued function on $[-1,1]$, so has an expansion $a_{i j}\left(s, s^{\prime}, T\right) \phi_{i}\left\langle\cdot, \phi_{j}\right\rangle$. Reinterpreting the $\phi_{i}$ as sections over $H$, and lifting to $B_{2}^{\infty}$ which is a blowup of $B_{2}\left(X_{\mathrm{Ls}}\right) \times[0, \infty]_{\sqrt{T}} \equiv([-1,0] \cup[0,1])_{s} \times H \times$ $[0, \infty]_{\sqrt{T}}$, the leading term at $B_{2}^{\infty}$ is (ias $\left.\epsilon\right) a_{i j}(s, s, T) \phi_{i}(y)\left\langle\cdot, \phi_{j}(y)\right\rangle$.

The restrictions in (7) are the data needed to compute the limit of analytic torsion under analytic surgery.

\section{Analytic and Reidemeister torsion.}

The analytic torsion of a flat unitary bundle $E$ over a manifold $M^{n}$ is defined via the zeta function of the Laplacian on $E$ :

$$
\begin{array}{r}
\log T\left(M^{n}, g, E\right)=\frac{1}{2} \sum_{q=0}^{n}(-1)^{q+1} q \log \operatorname{det} \Delta_{q}^{\prime} \equiv \frac{1}{2} \sum_{q=0}^{n}(-1)^{q} q \frac{d \zeta_{q}}{d s}(0) \\
\zeta_{q}(s)=\sum_{\substack{\lambda \in \operatorname{spec} \Delta_{q} \\
\lambda \neq 0}} \lambda^{-s}=\frac{1}{\Gamma(s)} \int_{0}^{\infty} t^{s} \operatorname{Tr} e^{-t \Delta_{q}^{\prime}} \frac{d t}{t}, \quad \operatorname{Re} s>\frac{n}{2}
\end{array}
$$

where $\zeta_{q}$ is the zeta function for $\Delta_{q}$, the Laplacian on $q$-forms with values in $E$, and $\Delta_{q}^{\prime}$ is $\Delta_{q}$ projected off the zero eigenspace. Using the heat kernel representation, one can show that the zeta function analytically continues to $s=0$. In order to generalize to b-analytic torsion, it is convenient to present this in a somewhat nonstandard way. Consider the two integrals

$$
\frac{1}{\Gamma(s)} \int_{0}^{C} F(t) \frac{d t}{t} \quad \text { and } \quad \frac{1}{\Gamma(s)} \int_{C}^{\infty} F(t) \frac{d t}{t}
$$

where $F(t)=t^{s} \operatorname{Tr} e^{-t \Delta_{q}}$. The first converges for Re $s$ large, and the second for $\operatorname{Re} s$ small. It is well known that the pointwise trace of the heat kernel on the diagonal has an expansion at $t=0$

$$
\operatorname{tr} e^{-t \Delta_{q}}(p, p)=\sum_{j \geq 0}^{K} t^{-\frac{n}{2}+j} a_{-\frac{n}{2}+j}\left(\Delta_{q}, g_{\epsilon}\right)(p)+O\left(t^{-\frac{n}{2}+K+1}\right)
$$

for any $K$, where the $a_{k}$ are universal polynomials involving the metric and its derivatives. Substituting this into the first integral in (9) shows that 
the integral meromorphically continues to $\mathbb{C}$. The trace of the heat kernel as $t \rightarrow \infty$ equals dim null $\Delta_{q}$ plus an exponentially decreasing part, so the second integral also meromorphically continues to $\mathbb{C}$ (in fact analytically, the zero in $1 / \Gamma$ at $s=0$ cancelling the pole coming from the constant part of $\operatorname{tr} e^{-t \Delta_{q}}$ at $t=\infty$ ). Therefore one can define the zeta function to be the sum of the meromorphic continuation of these two integrals.

From this one can derive a more explicit formula for analytic torsion, in the form of a convergent integral. The regularization (9) of $\int_{0}^{\infty} t^{z} d t / t$ is zero for every $z$. (That is why $F(t)$ was chosen to be $e^{-t \Delta_{q}}$ rather than $e^{-t \Delta_{q}^{\prime}}$.) Assume that $M$ is odd dimensional. Then the zeta function for $\Delta_{q}$ may be written in the form (9) with $F(t)$ equal to

$$
t^{s} \int_{M}\left\{\operatorname{tr} e^{-t \Delta_{q}}-\sum_{j=0}^{\frac{n-1}{2}} t^{-\frac{n}{2}+j} a_{-\frac{n}{2}+j}\left(M, \Delta_{q}, g_{\epsilon}\right)\right\} .
$$

The first integral then converges absolutely near $s=0$; the second will not because of the constant term projnull $\Delta_{q}$ in the trace of the heat kernel as $t \rightarrow \infty$. Pulling this term out and integrating yields an expression for the zeta function in terms of integrals absolutely convergent near $s=0$, which by differentiating in $s$ at $s=0$ gives the formula

$$
\begin{aligned}
& \log T\left(M, g_{\epsilon}\right)= \\
& =\frac{1}{2} \sum(-1)^{q} q\left[\int_{0}^{1} \frac{d t}{t} \int_{M}\left\{\operatorname{tr} e^{-t \Delta_{q}}-\sum_{j=0}^{\frac{n-1}{2}} t^{-\frac{n}{2}+j} a_{-\frac{n}{2}+j}\left(M, g_{\epsilon}\right)\right\}\right. \\
& \quad+\int_{1}^{\infty} \frac{d t}{t} \int_{M}\left\{\operatorname{tr} e^{-t \Delta_{q}^{\prime}}-\sum_{j=0}^{\frac{n-1}{2}} t^{-\frac{n}{2}+j} a_{-\frac{n}{2}+j}\left(M, g_{\epsilon}\right)\right\} \\
& \left.\quad+\gamma \operatorname{dim} \operatorname{null} \Delta_{q}\right]
\end{aligned}
$$

where $\gamma=\partial_{s} \Gamma(1)$ is Euler's constant.

All this goes over in a straightforward way to manifolds with boundary $N$ endowed with a b-metric. Consider the two integrals

$$
\frac{1}{\Gamma(s)} \int_{0}^{C} t^{s} b-\operatorname{Tr} e^{-t \Delta_{q, N}} \frac{d t}{t} \text { and } \frac{1}{\Gamma(s)} \int_{C}^{\infty} t^{s} b-\operatorname{Tr} e^{-t \Delta_{q, N}} \frac{d t}{t}
$$


The pointwise trace of the b-heat kernel has the same asymptotic expansion at $t=0$ as does the heat kernel on $M$ :

$$
\operatorname{tr} e^{-t \Delta_{q, N}}(p, p) \sim \sum_{j \geq 0}^{K} t^{-\frac{n}{2}+j} a_{-\frac{n}{2}+j}\left(\Delta_{q, N}, g_{\epsilon}, p\right)+O\left(t^{-\frac{n}{2}+K+1}\right)
$$

where the $a_{k}$ are as above. Hence the first integral is defined for Re $s$ large and meromorphically continues to $\mathbb{C}$. It is shown in [11] that the b-Trace of the heat kernel as $t \rightarrow \infty$ has an expansion in half-powers of $t$, so this integral converges for $\operatorname{Re} s \leq 0$ and continues meromorphically as before. Thus the b-zeta function may be defined by the meromorphic continuation of their sum:

$$
{ }^{b} \zeta_{q}(s)=\frac{1}{\Gamma(s)} \int_{0}^{C} t^{s} b-\operatorname{Tr} e^{-t \Delta_{q, N}} \frac{d t}{t}+\frac{1}{\Gamma(s)} \int_{C}^{\infty} t^{s} b-\operatorname{Tr} e^{-t \Delta_{q, N}} \frac{d t}{t} .
$$

The b-analytic torsion is defined by

$$
\log ^{b} T\left(N, g_{0}, E\right)=\frac{1}{2} \sum_{q=0}^{n}(-1)^{q} q \frac{d^{b} \zeta_{q}}{d s}(0)
$$

analogous to (8). Unlike the b-eta invariant of Part II, the spatial integrals defining b-analytic torsion are not absolutely convergent, and thus b-analytic torsion is sensitive to the boundary defining function used to compute the b-Trace. Recall from [11] that an exact b-metric determines a canonical section $|d x|$ of the conormal bundle of the boundary, up to a global constant multiple, by the condition that there are no terms $\frac{d x}{x} d y_{i}$ in the metric at the boundary. It is essential to take the b-Trace with respect to one of these canonical sections (the result is invariant under scaling by constants so the result is independent of the global constant multiple).

As with the analytic torsion, one can derive a formula for ${ }^{b} T$ in terms of convergent $t$-integrals. And just as with analytic torsion, the limit of the b-Trace of the heat kernel enters into the formula. Unfortunately this value was calculated incorrectly in [11]. In an appendix to this paper, the correct value is computed. Referring to (40) for the term proportional to $\gamma$, the 
formula is

$$
\begin{aligned}
& \log ^{b} T\left(N, g_{0}\right)= \\
& =\frac{1}{2} \sum(-1)^{q} q\left[\int_{0}^{1} \frac{d t}{t} b \int\left\{\operatorname{tr} e^{-t \Delta_{q}}-\sum_{j=0}^{\frac{n-1}{2}} t^{-\frac{n}{2}+j} a_{-\frac{n}{2}+j}\left(N, \Delta_{q}, g_{0}\right)\right\}\right. \\
& +\int_{1}^{\infty} \frac{d t}{t} b \int\left\{\operatorname{tr} e^{-t \Delta_{q}^{\prime}}-\sum_{j=0}^{\frac{n-1}{2}} t^{-\frac{n}{2}+j} a_{-\frac{n}{2}+j}\left(N, \Delta_{q}, g_{0}\right)\right\} \\
& \left.+\gamma \operatorname{dim} L^{2} \operatorname{null} \Delta_{q}\right] .
\end{aligned}
$$

If $M$ is even dimensional, then, as in the boundaryless case, the combination $\sum(-1)^{q} q^{b} \zeta_{q}(s)$ vanishes, and therefore the log of b-analytic torsion vanishes. To see this, note that in this case

$$
\begin{aligned}
\sum(-1)^{q} q \frac{1}{\Gamma(s)} \int_{A}^{B} t^{s} b-\operatorname{Tr} e^{-t \Delta_{q}} \frac{d t}{t} & = \\
& =\frac{n}{2} \frac{1}{\Gamma(s)} \int_{A}^{B} t^{s} b-\operatorname{Tr} \sum(-1)^{q} e^{-t \Delta_{q}} \frac{d t}{t} .
\end{aligned}
$$

By the APS index theorem for $d+\delta$, the b-Trace of $\sum(-1)^{q} e^{-t \Delta_{q}}$ is constant in $t$, equal to the Euler characteristic of $N$. (There is no boundary term in this case - see [11, chapter 9].) This makes the integrand a pure power of $t$, which is then regularized to zero.

The dependence of analytic torsion $T(M, g)$ on the metric $g$ can be described very simply. Let cohomology bases $\left\{\mu^{q}\right\} \subset H^{q}(M)$ be fixed. Using the Hodge theorem to identify $H^{q}(M)$ with harmonic forms, let $V^{q}$ be the volume of $\left\{\mu^{q}\right\}$ induced by the inner product on harmonic forms given by $g$. Then

$$
T(M, g)\left(\prod_{q \text { even }} V_{q}\right)^{-1}\left(\prod_{q \text { odd }} V_{q}\right)
$$

is independent of the metric [19]. Thus, $T$ can be thought of invariantly as a metric on the one-dimensional vector space

$$
\text { detline } H^{*}(M) \equiv \bigwedge^{*} H^{\text {even }}(M)\left(\bigwedge H^{\text {odd }}(M)\right)
$$


Here $\Lambda$ denotes the top exterior power of a vector space, and $\Lambda^{*}$ the top exterior power of its dual.

For computations in this paper, it is most convenient to study torsion relative to a fixed basis of cohomology classes - those which come from the 'Hodge Mayer-Vietoris sequence' for analytic surgery. Hence, we define, as in [19], analytic torsion with respect to a choice of bases $\left\{\mu^{i}\right\} \subset H^{i}(M)$ by

$$
T\left(M, \mu^{i}\right)=T(M, g) \prod_{q}\left[\omega^{q} \mid \mu^{q}\right]^{(-1)^{q}}
$$

where $\left\{\omega^{i}\right\}$ is an orthonormal basis of harmonic forms with respect to the metric $g$ and $\left[\omega^{q} \mid \mu^{q}\right]$ denotes the determinant of the change of basis matrix $W^{q}$, if $\omega^{q}=W^{q}\left(\mu^{q}\right)$. Equation (15) ensures that $T\left(M, \mu^{i}\right)$ is well defined, independent of the metric. In the setting of analytic surgery, $T\left(M, \mu^{i}\right)$ has the advantage of being independent of $\epsilon$.

Next, Reidemeister torsion (or R-torsion) is discussed. Let

$$
0 \rightarrow V^{0} \stackrel{d^{0}}{\longrightarrow} V^{1} \stackrel{d^{1}}{\longrightarrow} V^{2} \stackrel{d^{2}}{\longrightarrow} \ldots
$$

be a complex of finite dimensional vector spaces with inner product. Suppose preferred bases $\left\{\mu^{i}\right\} \subset H^{i}$ in cohomology are given. The torsion is an alternating product of determinants defined as follows. Let $B^{i} \subset V^{i}$ be the image of $d^{i-1}$ and let $b^{i}$ be a basis of $B^{i}$. Let $c^{i}$ denote an orthonormal basis of $V^{i}$. Using the notation [ | ] as above, the torsion is given by

$$
\tau\left(V, d, \mu^{i}\right)=\left|\prod_{q}\left[c^{q} \mid b^{q},\left(d^{q}\right)^{*}\left(b^{q+1}\right), \mu^{q}\right]^{(-1)^{q}}\right| .
$$

This definition is independent of the choice of $b^{i}$ as a change of basis will introduce into the product identical Jacobian factors in the numerator and denominator. Naturally, it does depend on the choice of volumes in cohomology. More invariantly, one can define the torsion as a metric on the determinant line of the cohomology of $V$. Note that many authors define torsion via volume elements $\omega^{* i}$ for $V^{i}$ and $\mu^{* i}$ for $H^{i}$. Since these lie in the dual spaces of $\Lambda V^{i}$ and $\Lambda H^{i}$, the formula for torsion then takes the form

$$
\tau=\left|\prod_{q}\left[\beta^{* q} \wedge\left(d^{q}\right)^{*} \beta^{* q+1} \wedge \mu^{* q} \mid \omega^{* q}\right]^{(-1)^{q}}\right|
$$

with $\beta^{* q} \in\left(\Lambda^{\operatorname{dim} B^{q}} V^{q}\right)^{*}, \beta^{* q}\left\lceil B^{q} \neq 0\right.$. Here it is written in the form (17) to make the comparison with (16) more evident. If the $\mu^{i}$ are taken to be 
an orthonormal basis of $H^{i}$ then $\tau$ has the alternate expression

$$
\tau=\prod_{q}\left|\operatorname{det} d_{\perp}^{q}\right|^{(-1)^{q}}
$$

where $d_{\perp}^{q}:\left(\operatorname{ker} d^{q}\right)^{\perp} \rightarrow \operatorname{im} d^{q}$ is the restriction of $d^{q}$. This formula is used in section 6 .

Given a simplicial decomposition of a manifold $M$, there is a cochain complex

$$
0 \rightarrow C^{0}(M) \stackrel{d^{0}}{\rightarrow} C^{1}(M) \stackrel{d^{1}}{\rightarrow} C^{2}(M) \stackrel{d^{2}}{\longrightarrow} \ldots
$$

whose elements are $\mathbb{R}$-valued (linear functionals on) formal sums of cells. Taking the inner product on $C^{q}$ that makes each cell orthonormal, and choosing volumes in cohomology $H^{*}(M)$, then a torsion is given by (17), called the R-torsion of $M$. The important property (see [14]) of this quantity is that the torsion is invariant under subdivision of the cells comprising $M$, and is therefore a topological invariant of $\left(M, \mu^{i}\right)$. One can also take a flat unitary bundle $E$ over $M$ and form the complex of cochains with values in $E$. Then (17) defines a topological invariant $\tau\left(M, E, \mu^{i}\right)$. From here on, a fixed flat unitary bundle $E$ over $M$ will be understood; it will usually be dropped from notation.

Notice that, with cohomology bases given by an orthonormal basis of harmonic forms with respect to some metric $g$ on $M$, the R-torsion has the same dependence on the metric as does the analytic torsion. With such a choice of bases, the torsion is denoted $\tau(M, E, g)$. Analytic and Reidemeister torsion share two other important properties: they vanish in even dimensions on closed manifolds, and they obey the same formula for the torsion of a product. These formal similarities led Ray and Singer to conjecture in [18] that the analytic and R-torsions of a closed manifold were equal.

If $M$ has boundary, there are a priori (at least) two R-torsions to consider, corresponding to the choice of relative or absolute cohomology. These are related by Poincare duality; if $M$ is odd dimensional, then the two are equal, so will simply be denoted $\tau(M)$.

\section{A Hodge Mayer-Vietoris sequence.}

In this section the behaviour of cohomology of flat unitary bundles under surgery is investigated. Some of the results here - in particular the counting argument below (20) - appeared previously in the preprint [4] of Cappell, Lee and Miller. 
Since $E$ is a flat bundle, the operator $d$ on $E$-valued forms determined by the (flat) connection on $E$ forms a twisted complex

$$
0 \rightarrow \Omega^{0}(M ; E) \rightarrow \Omega^{1}(M ; E) \rightarrow \Omega^{2}(M ; E) \rightarrow \ldots
$$

giving twisted cohomology groups $H^{q}(M ; E)$. If $M$ is the union of two open sets $U, V$ then there is a long exact sequence in cohomology, the MayerVietoris sequence:

$$
\begin{aligned}
\ldots \rightarrow H^{q-1}(U \cap V ; E) \rightarrow H^{q}(M ; E) \rightarrow & H^{q}(U ; E) \oplus H^{q}(V ; E) \rightarrow \\
& \rightarrow H^{q}(U \cap V ; E) \rightarrow \ldots
\end{aligned}
$$

If $H$ splits $M$ into two pieces $M_{ \pm}$then thickening each piece $M_{ \pm}$a little across $H$ yields two open sets to which Mayer-Vietoris can be applied; the intersection $U \cap V$ then is $H \times(-\delta, \delta)$ whose (absolute) cohomology is naturally the same as that of $H$. If $Z$ is a manifold with boundary, denote by $H_{\text {abs }}^{*}(Z), H_{\text {rel }}^{*}(Z)$ the cohomology, and the cohomology relative to the boundary respectively. Then the Mayer-Vietoris sequence takes the form

$$
\rightarrow H^{q-1}(H) \rightarrow H^{q}(M) \stackrel{j^{q}}{\longrightarrow} H_{\mathrm{abs}}^{q}\left(M_{+}\right) \oplus H_{\mathrm{abs}}^{q}\left(M_{-}\right) \stackrel{k^{q}}{\longrightarrow} H^{q}(H) \rightarrow
$$

(the bundle $E$ is understood and dropped from the notation, here and below).

On a compact manifold $M$ without boundary, the Hodge Theorem states that the space of harmonic forms with respect to any metric is isomorphic to the de Rham cohomology and gives canonical choices (given the metric) for cohomology classes on $M$. It is useful to have canonical choices of cohomology for all spaces in the exact sequence (19) for the computations in the next chapter. For this purpose a Hodge version of this exact sequence is developed.

As a first step towards a Hodge version of the sequence (19), recall results from [11, chapter 6] on the Hodge theory of a manifold $Z$ with boundary and exact b-metric. This 'b-Hodge theory' works equally well with twisted cohomology groups corresponding to a flat unitary bundle. In [11] relative and absolute Hodge cohomology groups $H_{b \text {-abs, } \mathrm{Ho}}^{*}(Z)$ and $H_{b \text {-rel,Ho }}^{*}(Z)$ are defined, being bounded elements of the null space of $\Delta_{Z}$. Forms $\alpha \in H_{b \text {-abs, } \mathrm{Ho}}^{*}(Z)$ and $\beta \in H_{b \text {-rel,Ho }}^{*}(Z)$ are characterized by their behaviour near $\partial Z$. In product coordinates $(x, y)$ near $\partial Z$,

$$
\begin{aligned}
& \alpha=\alpha^{\prime}(y)+O\left(x^{\delta}\right) \\
& \beta=\frac{d x}{x} \wedge \beta^{\prime}(y)+O\left(x^{\delta}\right) \quad \text { near } \partial Z \text { for some } \delta>0 .
\end{aligned}
$$


The boundary-data map $\mathrm{BD}^{*}$ is defined by $\mathrm{BD}^{*}(\alpha)=\alpha^{\prime}, \mathrm{BD}^{*}(\beta)=\beta^{\prime}$. The null space of $\mathrm{BD}^{*}$, consisting of forms which are $O\left(x^{\delta}\right)$ near $\partial Z$, is the $L^{2}$ cohomology space $H_{b \text {-Ho }}^{q}(Z)$. The groups have an inner product given by the $L^{2}$ norm on $L^{2}$ cohomology and the inner product induced from $H^{q}(H)$ by $\mathrm{BD}^{*}$. The boundary-data map is such that $\mathrm{BD}^{q}\left(H_{b \text {-abs,Ho }}^{q}(Z)\right) \oplus$ $\mathrm{BD}^{q}\left(H_{b-\text { rel,Ho }}^{q+1}(Z)\right)$ is an orthogonal decomposition of $H^{q}(\partial Z)$; thus, these groups can be assembled into a relative cohomology sequence

$$
\begin{aligned}
\rightarrow H_{b-\mathrm{abs}, \mathrm{Ho}}^{q}(Z) \stackrel{\mathrm{BD}^{q}}{\longrightarrow} H^{q}(\partial Z) \stackrel{\left(\mathrm{BD}^{q}\right)^{*}}{\longrightarrow} H_{b-\mathrm{rel}, \mathrm{Ho}}^{q+1}(Z) & \rightarrow \\
& \rightarrow H_{b-\mathrm{abs}, \mathrm{Ho}}^{q+1}(Z) \rightarrow .
\end{aligned}
$$

The unlabelled map is the identity on the $L^{2}$ cohomology and is zero on its orthocomplement.

Denote the images $\mathrm{BD}^{q}\left(H_{b-\mathrm{abs}, \mathrm{Ho}}^{q}\left(M_{ \pm}\right)\right)$and $\mathrm{BD}^{q}\left(H_{b \text {-rel,Ho}}^{q+1}\left(M_{ \pm}\right)\right) \subset$ $H^{q}(H)$ by $A_{ \pm}^{q}, R_{ \pm}^{q}$ respectively. Then $A_{ \pm}^{q}=\left(R_{ \pm}^{q}\right)^{\perp}=* R_{ \pm}^{n-1-q}$ (Poincaré duality), where $\operatorname{dim} M=n$.

Consider the implications of b-Hodge theory for the sequence (19) under surgery. By exactness of (19),

$$
\begin{aligned}
H^{q}(M) & \equiv \operatorname{im}\left(H^{q-1}(H) \rightarrow H^{q}(M)\right) \oplus \operatorname{im} j^{q} \\
& \equiv\left(\operatorname{im} k^{q-1}\right)^{\perp} \oplus \operatorname{ker} k^{q} .
\end{aligned}
$$

By the remarks above,

$$
\left(\mathrm{im} k^{q-1}\right)^{\perp}=\left(A_{+}^{q-1}+A_{-}^{q-1}\right)^{\perp}=R_{+}^{q-1} \cap R_{-}^{q-1}
$$

and

$$
\operatorname{ker} k^{q}=A_{+}^{q} \cap A_{-}^{q} \oplus H_{b-\mathrm{Ho}}^{q}\left(M_{+}\right) \oplus H_{b-\mathrm{Ho}}^{q}\left(M_{-}\right) \text {. }
$$

Hence,

$$
\begin{aligned}
\operatorname{dim} H^{q}(M)=\operatorname{dim}\left(R_{+}^{q-1} \cap R_{-}^{q-1}\right) & +\operatorname{dim}\left(A_{+}^{q} \cap A_{-}^{q}\right) \\
& +\operatorname{dim}\left(H_{b-\mathrm{Ho}}^{q}\left(M_{+}\right) \oplus H_{b-\mathrm{Ho}}^{q}\left(M_{-}\right)\right) .
\end{aligned}
$$

Now recall the results on small eigenvalues proved in chapter 6 of Part II. It was shown that the eigenvalues $z(\epsilon)$ are continuous down to $\epsilon=0$, with limiting behaviour (ias $\epsilon)^{2} z^{2}+o\left((\operatorname{ias} \epsilon)^{2}\right)$, where $z=0$ (corresponding to $L^{2}$ null space) or $z^{2}=z_{j}^{2} \in \operatorname{spec} \operatorname{RN}\left(\Delta_{q}\right)$. It is not hard to check that the multiplicity of $0 \in \operatorname{spec} \operatorname{RN}(\Delta)$ is the dimension of the intersection $\Lambda_{+}^{D} \cap$ $\Lambda_{-}^{D}$. The intersection $\Lambda_{+}^{D} \cap \Lambda_{-}^{D}$ is, for the Laplacian on $q$-forms, equal to 
$A_{+}^{q} \cap A_{-}^{q} \oplus R_{+}^{q-1} \cap R_{-}^{q-1}$, so the dimension of the space of eigenfunctions corresponding to $z=0$ is

$$
\operatorname{dim} H_{b-\mathrm{Ho}}^{q}\left(M_{+}\right)+\operatorname{dim} H_{b-\mathrm{Ho}_{\mathrm{o}}}^{q}\left(M_{-}\right)+\operatorname{dim}\left(A_{+}^{q} \cap A_{-}^{q}\right)+\operatorname{dim}\left(R_{+}^{q-1} \cap R_{-}^{q-1}\right) .
$$

The cohomology $H^{q}(M)$ is, by ordinary Hodge theory, given for each $\epsilon>0$ by the null space of $\Delta_{q}$, so comparing (20) and (21) shows that all the eigenforms corresponding to $z=0$ represent cohomology on $M$ and therefore all eigenvalues corresponding to $z=0$ remain identically zero for $\epsilon>0$. In other words, the multiplicity of $0 \in \operatorname{spec} \Delta^{q}$ is constant as $\epsilon \downarrow 0$. It confirms the intuitively plausible idea that one ought to have a cohomology element on $M$ for every pair of elements on $M_{+}$and $M_{-}$that match at $H$.

This fact has regularity implications for the generalized inverse of $\Delta_{\epsilon}$. Let $\Pi_{\epsilon}$, for each $\epsilon>0$, be the projection onto the null space of $\Delta_{\epsilon}$.

Proposition 5. (1) The Schwartz kernel of the projection $\Pi_{\epsilon}$ is smooth down to ias $\epsilon=0$ on $X_{\mathrm{Ls}}^{2}$.

(2) The full heat kernel $e^{-t \Delta_{\epsilon}}$ on the diagonal is smooth on $\Delta_{\mathrm{LHs}}$ at $t=\infty$. (Compare Theorem 45 of Part II.)

Proof (Sketch). (1) In Part II, section 6 it is shown that the projection onto all eigenfunctions corresponding to one $z_{j}^{2} \in \operatorname{spec} \mathrm{RN}(\Delta)$ is in $\Psi^{-\infty,-1}\left(X_{\mathrm{Ls}}^{2} ; \Omega_{D}^{\frac{1}{2}} X_{\mathrm{Ls}}^{2}\right)$. In fact, the Schwartz kernel of $\Pi_{\epsilon}$ is easily seen to be bounded as ias $\epsilon \rightarrow 0$, so $\Pi_{\epsilon} \in \Psi^{-\infty, 0}\left(X_{\mathrm{Ls}}^{2} ; \Omega_{D}^{\frac{1}{2}} X_{\mathrm{Ls}}^{2}\right)$. In particular, $\Pi_{\epsilon}$ is smooth down to ias $\epsilon=0$.

(2) By Part II, Proposition 43, the heat kernel projected off null modes, $e^{-t \Delta_{\epsilon}^{\perp}}$ is smooth on $\Delta_{\text {LHs }}$ at $t=\infty$, and so the full heat kernel $e^{-t \Delta_{\epsilon}}=$ $e^{-t \Delta_{\epsilon}^{\perp}}+\Pi_{\epsilon}$ is also smooth.

The second property explains why there is no extra term depending on very small eigenvalues in the surgery formula for analytic torsion as there is for the eta invariant in Part II.

The range of $\Pi_{\epsilon}$ is called the surgery Hodge cohomology of $M, H_{s-\text { Ho }}^{*}(M)$. Using Proposition 5 the image in $H_{s-\mathrm{Ho}}^{*}(M)$ of a cohomology class $[\alpha]$, where $\alpha$ is a closed $E$-valued form on $M$, can be determined. Lifting $\alpha$ to $\tilde{\alpha}$ on $X_{\mathrm{Ls}}$, by the above proposition the image is given by $\Pi_{\epsilon}(\tilde{\alpha})$. To analyse the behaviour of $\Pi_{\epsilon} \tilde{\alpha}$ as $\epsilon \rightarrow 0$, let $\phi_{i}^{0}$ be an orthonormal basis for $H_{b-\mathrm{Ho}}^{*}\left(M_{+}\right) \oplus$ 
$H_{b-\mathrm{Ho}}^{*}\left(M_{-}\right)$, let

$\psi_{j,+}^{0}$ be an orthonormal basis for $\left\{\psi \in H_{b-\mathrm{abs}, \mathrm{Ho}}^{*}\left(M_{+}\right) \mid \mathrm{BD}(\psi) \in A_{-}^{*}\right\}$,

$\chi_{k,+}^{0}$ be an orthonormal basis for $\left\{\chi \in H_{b-\text { rel,Ho }}^{*}\left(M_{+}\right) \mid \mathrm{BD}(\psi) \in R_{-}^{*}\right\}$.

and let $\psi_{j,-}^{0}, \chi_{k,-}^{0}$ be the corresponding elements of $H_{b-\text { abs,Ho }}^{*}\left(M_{-}\right)$, $H_{b \text {-rel,Ho }}^{*}\left(M_{-}\right)$. The sections $\phi_{i}^{0}, \psi_{j, \pm}^{0}, \chi_{k, \pm}^{0}$ can be extended to smooth sections $\phi_{i}, \psi_{j}, \chi_{k}$ on $X_{\mathrm{Ls}}$ such that $\left(\phi_{i},(\text { ias } \epsilon)^{1 / 2} \psi_{j},(\text { ias } \epsilon)^{1 / 2} \chi_{k}\right)$ form an orthonormal basis of the range of $\Pi_{\epsilon}$. (They can be constructed conormal on $X_{s}$ and are then smooth when lifted to $X_{\mathrm{Ls}}$.) Near $H, \alpha$ has the form $\alpha_{a}+\alpha_{r} d x$ in product coordinates, where $\alpha_{a}, \alpha_{r}$ are closed forms on $H$. At $\epsilon=0$ near $H$, in terms of surgery forms (see Part II, section 3) $\tilde{\alpha}=\alpha_{a}+\sqrt{x^{2}+\epsilon^{2}} \alpha_{r}\left(d x / \sqrt{x^{2}+\epsilon^{2}}\right)$. Then

$$
\Pi_{\epsilon} \tilde{\alpha}=\phi_{i}\left\langle\tilde{\alpha}, \phi_{i}\right\rangle+(\operatorname{ias} \epsilon) \psi_{j}\left\langle\tilde{\alpha}, \psi_{j}\right\rangle+(\operatorname{ias} \epsilon) \chi_{k}\left\langle\tilde{\alpha}, \chi_{k}\right\rangle .
$$

Because the coefficient of $d x / \sqrt{x^{2}+\epsilon^{2}}$ vanishes at $B_{1}$ and $B_{2}$,

$$
\begin{aligned}
\left\langle\tilde{\alpha}, \phi_{i}\right\rangle & =a_{i}(\operatorname{ias} \epsilon) \\
\left\langle\tilde{\alpha}, \psi_{j}\right\rangle & =(\operatorname{ias} \epsilon)^{-1} b_{j}(\operatorname{ias} \epsilon) \\
\left\langle\tilde{\alpha}, \chi_{k}\right\rangle & =c_{k}(\operatorname{ias} \epsilon),
\end{aligned}
$$

where $a_{i}, b_{j}, c_{k}$ are smooth functions of ias $\epsilon$. Hence

$$
\Pi_{\epsilon} \tilde{\alpha}=a_{i} \phi_{i}+b_{j} \psi_{j}+(\operatorname{ias} \epsilon) c_{k} \chi_{k},
$$

with $\left(a_{i}, b_{j}, c_{k}\right)$ smooth functions of $\epsilon$, linearly independent as $[\alpha]$ ranges over $H^{*}(M)$. Define an inner product on $H_{s-\mathrm{Ho}}^{*}(M)$ by setting

$$
\left|\Pi_{\epsilon} \tilde{\alpha}\right|^{2}=\sum_{i}\left|a_{i}(0)\right|^{2}+2 \sum_{j}\left|b_{j}(0)\right|^{2}+2 \sum_{k}\left|c_{k}(0)\right|^{2} .
$$

This is independent of the choice of orthonormal vectors $\phi_{i}, \psi_{j}, \chi_{k}$. Hence $H_{s-\mathrm{Ho}}^{*}(M)$ splits into three orthogonal subspaces:

$$
\begin{aligned}
& H_{s-L^{2}, \mathrm{Ho}}^{*}(M)=\left\{\Pi_{\epsilon} \tilde{\alpha} \mid b_{j}(0)=c_{k}(0)=0\right\} ; \\
& H_{s \text {-abs, } \mathrm{Ho}}^{*}(M)=\left\{\Pi_{\epsilon} \tilde{\alpha} \mid a_{i}(0)=c_{k}(0)=0\right\} ; \\
& H_{s \text {-rel,Ho }}^{*}(M)=\left\{\Pi_{\epsilon} \tilde{\alpha} \mid a_{i}(0)=b_{j}(0)=0\right\} .
\end{aligned}
$$

Note that the $L^{2}$ norms of elements of these three subspaces have the leading behaviours (ias $\epsilon)^{k}$ where $k=0,-\frac{1}{2}, \frac{1}{2}$ respectively. In the Mayer-Vietoris sequence (19) $H_{s \text {-rel,Ho }}^{q}(M)$ is the image of the connecting homomorphism 
$H^{q-1}(H) \rightarrow H^{q}(M)$ and the map $j^{q}$ on $H_{s-\mathrm{Ho}}^{q}(M)$ is restriction to the boundary $B_{0}$. With the inner product (23), both these maps are isometries from the orthocomplement of the preceding map to their image.

The b-analytic torsion obeys a formula analogous to (15) under deformations of the metric. In fact, if $V_{q}$ denotes the volumes of fixed elements of either relative or absolute cohomology with respect to the inner product defined by b-Hodge theory, then

$$
{ }^{b} T(N, g)=\left(\prod_{q \text { even }} V_{q}\right)\left(\prod_{q \text { odd }} V_{q}\right)^{-1} \overline{{ }^{b} T}(N),
$$

where $\bar{b} \bar{T}$ is independent of the metric. This result is only used below to avoid assuming that the metric on $N$ in Theorem 2 is an exact product near the boundary, so the details of proof are omitted. It follows from the same formal manipulations which are used to prove (15) in [19]. In these manipulations, one needs to compute the b-Trace of certain commutators; this can be done using the formula from [11, chapter 5]. All such commutators turn out to vanish, making the final result very similar to the boundaryless case.

\section{Surgery formula for analytic torsion.}

The surgery formula for analytic torsion is derived by performing the integral (10). As the integrand lies on a blown-up version of its natural domain, the integrand is really a pushforward from $\Delta_{\mathrm{LHs}}$ to $\left[0, \operatorname{ilg} \epsilon_{0}\right]_{\mathrm{ilg} \epsilon}$. To compute the result, the Pushforward Theorem of [12] is applied. The general form of the computation is treated in Part II, Lemma 9. From this computation the formula (30) below is obtained relating $T(M),{ }^{b} T(\bar{M})$ and the log determinants of $\operatorname{RN}\left(\Delta_{q}\right)$. Comparing to a surgery formula for R-torsion, Theorem 1 is obtained.

Step 1. As a first step, consider the calculation of the log determinant of the Laplacian on the interval $[-1,1]_{x}$ with boundary conditions $(6)$ under surgery. This is useful both as a model calculation for analytic torsion, and because it involves a calculation essential in Step 2. The log determinant can be calculated in two ways: using the exact scaling property of the Laplacian on an interval under surgery, and as a pushforward. In Part II, section 5, it is shown that the reduced normal operator of this operator under surgery reproduces the original operator. The interval $[-1,1]_{x}$ with surgery metric $g_{\epsilon}=d x^{2} /\left(x^{2}+\epsilon^{2}\right)$ has length $2 \sinh ^{-1}(x / \epsilon)=2(\operatorname{ias} \epsilon)^{-1}$, so the zeta function 
for the Laplacian corresponding to $g_{\epsilon}$ satisfies

$$
\zeta(s, \epsilon)=(\operatorname{ias} \epsilon)^{-2 s} \zeta(s)
$$

where $\zeta(s)$ is the zeta function of $\operatorname{RN}(\Delta)$, and so

$$
\log \operatorname{det} \zeta(\epsilon)=-2 \log (\operatorname{ias} \epsilon) \operatorname{dim} \operatorname{null} \mathrm{RN}(\Delta)+\log \operatorname{det} \mathrm{RN}(\Delta) .
$$

Calculating the second way, the zeta function has an expression

$$
\begin{aligned}
\zeta(s, \epsilon)= & \frac{1}{\Gamma(s)} \int_{0}^{1} t^{s}\left(\operatorname{Tr} e^{-t \Delta_{\epsilon}}-\frac{2}{\sqrt{4 \pi t}}\right) \frac{d t}{t} \\
& +\frac{1}{\Gamma(s)} \int_{1}^{\infty} t^{s}\left(\operatorname{Tr} e^{-t \Delta_{\epsilon}^{\prime}}-\frac{2}{\sqrt{4 \pi t}}\right) \frac{d t}{t}-\frac{1}{s \Gamma(s)} \operatorname{dim} \operatorname{null} \mathrm{RN}(\Delta)
\end{aligned}
$$

near $s=0$. Thus $\zeta(0, \epsilon)=-\operatorname{dim} \operatorname{null} \mathrm{RN}(\Delta)$, and

$$
\begin{aligned}
\log \operatorname{det} \Delta_{\epsilon}=-\zeta^{\prime} & (0, \epsilon)=\int_{0}^{1}\left(\operatorname{Tr} e^{-t \Delta_{\epsilon}}-\frac{2}{\sqrt{4 \pi t}}\right) \frac{d t}{t} \\
& +\int_{1}^{\infty}\left(\operatorname{Tr} e^{-t \Delta_{\epsilon}^{\prime}}-\frac{2}{\sqrt{4 \pi t}}\right) \frac{d t}{t}-\gamma \operatorname{dim} \operatorname{null} \mathrm{RN}(\Delta)
\end{aligned}
$$

with $\gamma$ again Euler's constant. Computing this as a pushforward, it follows from Lemma 9 of Part II that $\log \operatorname{det} \Delta_{\epsilon}$ has an expansion

$$
\log \operatorname{det}\left(\Delta, g_{\epsilon}\right) \sim \sum_{(n, k) \in \mathcal{E}} b_{n, k}(\operatorname{ias} \epsilon)^{n}(\log (\operatorname{ias} \epsilon))^{k} .
$$

where $\mathcal{E}$ is the index set

$$
\mathcal{E}=\{(-1,0)\} \cup\{(n, 0),(n, 1) \mid n \in \mathbb{N}\} .
$$

The first computation shows that only $b_{0,1}$ and $b_{0,0}$ are nonzero. The term $b_{0,1}$ is given by the integral (25) restricted to $B_{2}^{\infty} \cap B_{0}$ and $B_{2}^{\infty} \cap B_{1}$ (there is no contribution from $B_{2} \cap B_{0}$ or $B_{2} \cap B_{1}$ because the heat kernel on the diagonal for finite time can be lifted from $X_{s} \times[0, C]_{\sqrt{t}}$ and there can be no $\log$ (ias $\epsilon$ ) term from this space). The $b_{0,0}$ term is the sum of b-integrals on $B_{0}$ and $B_{1}$ and on $B_{2}^{\infty}$. On $B_{0}$ and $B_{1}$, where are metrically copies of $\mathbb{R}$, the Laplacian is translation invariant, and so the b-integrals are zero. Hence, using the fourth line of (7),

$$
b_{0,0}=\log \operatorname{det}(\mathrm{RN}(\Delta))=\gamma \operatorname{dim} \operatorname{null} \mathrm{RN}(\Delta)+{ }^{b} \int_{B_{2}^{\infty}} \operatorname{tr} e^{-T \mathrm{RN}(\Delta)} .
$$


Step 2. In this step the result of the calculation in Step 1 is used in the main computation, which is the surgery formula for analytic torsion. Let $p_{[0,1]}\left(p_{[1, \infty]}\right)$ be the stretched projection from $\Delta_{\mathrm{LHs}}^{[0,1]}\left(\Delta_{\mathrm{LHs}}^{[1, \infty]}\right)$ to $\left[0, \operatorname{ilg} \epsilon_{0}\right]_{\epsilon}$. From (10), analytic torsion can be written as a pushforward

$$
\begin{aligned}
& =\sum_{q=0}^{n} \frac{(-1)^{q} q}{2}\left\{p_{[0,1]_{*}}\left(\operatorname{tr} e^{-t \Delta_{q}}-\sum_{j=0}^{\frac{n-1}{2}} t^{-\frac{n}{2}+j} a_{-\frac{n}{2}+j}\left(M, g_{\epsilon}\right)\right)\left|\frac{d(\operatorname{ilg} \epsilon)}{(\operatorname{ilg} \epsilon)^{2}}\right|^{-1}\right. \\
& +p_{[1, \infty]_{*}}\left(\operatorname{tr} e^{-t \Delta_{q}^{\prime}}-\sum_{j=0}^{\frac{n-1}{2}} t^{-\frac{n}{2}+j} a_{-\frac{n}{2}+j}\left(M, g_{\epsilon}\right)\right) \cdot\left|\frac{d(\operatorname{ilg} \epsilon)}{(\operatorname{ilg} \epsilon)^{2}}\right|^{-1} \\
& \left.+\gamma \operatorname{dim} \text { null } \Delta_{q}\right\} .
\end{aligned}
$$

As with the log determinant above, there is an asymptotic expansion

$$
\log T\left(M, g_{\epsilon}\right) \sim \sum_{(n, k) \in \mathcal{E}} a_{n, k}(\operatorname{ias} \epsilon)^{n}(\log (\operatorname{ias} \epsilon))^{k} .
$$

where $\mathcal{E}$ is the index set

$$
\mathcal{E}=\{(-1,0)\} \cup\{(n, 0),(n, 1) \mid n \in \mathbb{N}\} .
$$

The actual object of interest is the torsion with respect to fixed cohomology classes

$$
\log T\left(M, \mu^{i}\right)=\log T\left(M, g_{\epsilon}\right)+\sum(-1)^{q} \log \left[\omega^{q} \mid \mu^{q}\right] .
$$

Choose cohomology classes $\mu^{q}$ corresponding to an orthonormal basis of surgery Hodge cohomology as defined by (22). Then the determinant factors in (29) are of the form

$$
\left((\operatorname{ias} \epsilon)^{-\frac{1}{2}}+O(1)\right)^{ \pm 1} \quad \text { or } 1+O(\operatorname{ias} \epsilon)
$$

The sum of $\log$ arithms of these therefore has the form $C \cdot \log (\operatorname{ias} \epsilon)+O(\operatorname{ias} \epsilon)$. This does not contain any constant term in ias $\epsilon$. Since it is known a priori that $\log T\left(M, \mu^{i}\right)$ is constant in ias $\epsilon$, it follows that $\log T\left(M, \mu^{i}\right)$ is equal to $a_{0,0}$ in (28). Hence it is only necessary to compute $a_{0,0}$. By Lemma 9 from Part II, this comes from b-integrals on $B_{1}, B_{0}$ and $B_{2}^{\infty}$. As in the 
model calculation above, the contribution from $B_{1}$ is zero, because the heat kernel $e^{-t \Delta_{H \times \mathbb{R}}}$ is translation invariant in the $\mathbb{R}$-direction and so its b-trace vanishes identically. From $B_{0}$, comparing (10) and (14) shows that the contribution is precisely the b-analytic torsion of $\bar{M}$ plus $\gamma\left(\operatorname{dim}\right.$ null $\Delta_{q, M}-$ $\operatorname{dim} L^{2}$ null $\left.\Delta_{q, \bar{M}}\right)$. Finally, from $B_{2}^{\infty}$, the contribution is the same as in (26) because by $(7)$ the restriction of the heat kernel on this face is the heat kernel of the reduced normal operator. Therefore,

$$
\begin{aligned}
\log T\left(M, \mu^{i}\right)= & a_{0,0}=\log ^{b} T\left(\bar{M}, g_{0}\right)+\frac{1}{2} \sum_{q=0}^{n}(-1)^{q} q\left[\log \operatorname{det} \mathrm{RN}\left(\Delta_{q}\right)\right. \\
& \left.+\gamma\left(\operatorname{dim} \operatorname{null} \Delta_{q, M}-\operatorname{dim} L^{2} \operatorname{null} \Delta_{q, \bar{M}}-\operatorname{dim} \operatorname{null} \mathrm{RN}\left(\Delta_{q}\right)\right)\right] .
\end{aligned}
$$

But (20), the Hodge Theorem, and the fact that $\operatorname{dim}$ null $\mathrm{RN}\left(\Delta_{q}\right)=$ $\operatorname{dim} \Lambda_{+}^{D} \cap \Lambda_{-}^{D}=\operatorname{dim} A_{+}^{q} \cap A_{-}^{q}+\operatorname{dim} R_{+}^{q} \cap R_{-}^{q}$ show that the coefficient of $\gamma$ is zero. Hence this reduces to

$$
\log T\left(M, \mu^{i}\right)=\log ^{b} T\left(\bar{M}, g_{0}\right)+\frac{1}{2} \sum_{q=0}^{n}(-1)^{q} q \log \operatorname{det} \operatorname{RN}\left(\Delta_{q}\right) .
$$

Step 3. The next step is to find an explicit formula for $\log \operatorname{det} \mathrm{RN}(\Delta)$ in terms of the subspaces $\Lambda_{ \pm}^{D}, \Lambda_{ \pm}^{N}$ that determine it. To state this result, first decompose the vector space $V=$ null $\Delta_{H}$ into an orthogonal direct sum $V=V_{1} \oplus V_{2} \oplus V_{3}$, where

$$
\begin{aligned}
& V_{1}=\Lambda_{+}^{D} \cap \Lambda_{-}^{D} \oplus \Lambda_{+}^{N} \cap \Lambda_{-}^{N} \\
& V_{2}=\Lambda_{+}^{D} \cap \Lambda_{-}^{N} \oplus \Lambda_{-}^{D} \cap \Lambda_{+}^{N} \\
& V_{3}=V \ominus\left(V_{1} \oplus V_{2}\right) .
\end{aligned}
$$

Write $\Lambda_{ \pm}^{D, r}, \Lambda_{ \pm}^{N, r}$ for the 'reduced' subspaces $\Lambda_{ \pm}^{D} \cap V_{3}, \Lambda_{ \pm}^{N} \cap V_{3}$, two pairs of orthogonal complements in $V_{3}$ all of which intersect only in $\{0\}$, and write $S_{ \pm}^{r}$ for the scattering matrices $S_{ \pm}(0)$ restricted to $V_{3}$. The Laplacian $\Delta$ splits into a direct sum $\Delta=\Delta_{1}+\Delta_{2}+\Delta_{3}$ with $\Delta_{i}$ acting on sections of $V_{i}$, and so $\log \operatorname{det} \Delta=\log \operatorname{det} \Delta_{1}+\log \operatorname{det} \Delta_{2}+\log \operatorname{det} \Delta_{3}$.

Proposition 6. The log determinants of $\Delta_{1}, \Delta_{2}, \Delta_{3}$ are given by

$$
\begin{aligned}
& \log \operatorname{det} \Delta_{1}=2 \log 2 \operatorname{dim} V_{1} \\
& \log \operatorname{det} \Delta_{2}=\log 2 \operatorname{dim} V_{2} \\
& \log \operatorname{det} \Delta_{3}=\log \operatorname{det}\left(\operatorname{Id}-S_{+}^{r} S_{-}^{r}\right) .
\end{aligned}
$$


Proof. The operator $\Delta_{1}$ has nonzero eigenvalues $\left(\frac{\pi k}{2}\right)^{2}, k \geq 1$, with multiplicity $\operatorname{dim} V_{1}$ and the operator $\Delta_{2}$ has eigenvalues $\left(\frac{\pi(k-1 / 2)}{2}\right)^{2}, k \geq 1$, with multiplicity $\operatorname{dim} V_{2}$. The $\log$ determinant of these two operators can be calculated from the values of the Riemann zeta function and its derivative at $s=0$; the result is (31) and (32).

To calculate $\log \operatorname{det} V_{3}$, recall from Part II, section 5 that the eigenvalues of $\operatorname{RN}\left(\Delta_{3}\right)$ are periodic with period $\pi / 2$. It is well known that the $\log$ determinant of an operator with eigenvalues $(n \pi / 2+\alpha)^{2}$ is $2 \log (2 \sin 2 \alpha)$, for $\alpha \neq k \pi / 2$. Note that $\Delta_{3}$ has no zero eigenvalues, because the multiplicity of 0 as an eigenvalue is precisely $\operatorname{dim} \Lambda_{+}^{D, r} \cap \Lambda_{-}^{D, r}$. Therefore the log determinant is the sum

$$
\sum_{j} 2 \log 2 \sin 2 \alpha_{j}
$$

over eigenvalues $\alpha_{j}$ such that $0<\alpha_{j}<\pi / 2$.

If $\alpha \in(0, \pi / 2)$ is an eigenvalue of $\Delta_{3}$, then the corresponding eigenfunction may be written

$$
e^{i \alpha s} \phi+e^{-i \alpha s} \psi
$$

The boundary conditions imply that

$$
\begin{aligned}
\phi+e^{-2 i \alpha} \psi \in \Lambda_{+}^{D, r}, & \phi-e^{-2 i \alpha} \psi \in \Lambda_{+}^{N, r}, \\
\phi+e^{2 i \alpha} \psi \in \Lambda_{-}^{D, r}, & \phi-e^{2 i \alpha} \psi \in \Lambda_{-}^{N, r} .
\end{aligned}
$$

From this follows

$$
\begin{array}{ll}
\operatorname{proj} \Lambda_{+}^{D, r} \phi=\operatorname{proj} \Lambda_{+}^{D, r} e^{-2 i \alpha} \psi, & \operatorname{proj} \Lambda_{-}^{D, r} \phi=\operatorname{proj} \Lambda_{-}^{D, r} e^{2 i \alpha} \psi, \\
\operatorname{proj} \Lambda_{+}^{N, r} \phi=-\operatorname{proj} \Lambda_{+}^{N, r} e^{-2 i \alpha} \psi, & \operatorname{proj} \Lambda_{-}^{N, r} \phi=-\operatorname{proj} \Lambda_{-}^{N, r} e^{2 i \alpha} \psi,
\end{array}
$$

whence, by (4),

$$
\begin{array}{ll}
\phi=e^{-2 i \alpha} S_{+}^{r} \psi, & S_{+}^{r} S_{-}^{r} \phi=e^{4 i \alpha} \phi, \\
\phi=e^{2 i \alpha} S_{-}^{r} \psi, & S_{+}^{r} S_{-}^{r} \psi=e^{-4 i \alpha} \psi .
\end{array}
$$

By Weyl's Law and the $\pi / 2$-periodicity of eigenvalues of $\Delta_{3}$, it follows that $\alpha \in(0, \pi / 2)$ is an eigenvalue of $\Delta_{3}$ if and only if both $e^{4 i \alpha}$ and $e^{-4 i \alpha}$ are 
eigenvalues of $S_{+}^{r} S_{-}^{r}$. Then

$$
\begin{aligned}
\log \operatorname{det}\left(\operatorname{Id}-S_{+}^{r} S_{-}^{r}\right) & =\log \prod_{j}\left(1-e^{4 i \alpha_{j}}\right)\left(1-e^{-4 i \alpha_{j}}\right) \\
& =\log \prod_{j}\left(2-2 \cos 4 \alpha_{j}\right) \\
& =\sum_{j} \log \left(4 \sin ^{2} 2 \alpha\right)=2 \sum_{j} \log (2 \sin 2 \alpha) .
\end{aligned}
$$

This agrees with (34), so (33) is established.

Step 4. Next substitute in (30) the results of Proposition 6 to get the surgery formula in terms of cohomology. In this case, $\Lambda_{ \pm}^{D}=A_{ \pm}^{q} \oplus R_{ \pm}^{q-1}$, $\Lambda_{ \pm}^{N}=R_{ \pm}^{q} \oplus A_{ \pm}^{q-1}$. Write $A_{ \pm}^{q, r}, R_{ \pm}^{q, r}$ for the reduced spaces analogous to $\Lambda_{ \pm}^{D, r}, \Lambda_{ \pm}^{N, r}$, and write

$$
S_{ \pm}^{q, r} \equiv \operatorname{proj} A_{ \pm}^{q, r}-\operatorname{proj} R_{ \pm}^{q, r},
$$

so that $S_{ \pm}^{r}\left(\Delta_{q}\right)=S_{ \pm}^{q, r} \oplus-S_{ \pm}^{q-1, r}$. Then

$$
\begin{aligned}
\frac{1}{2} \sum & (-1)^{q+1} q \log \operatorname{det} \mathrm{RN}\left(\Delta_{q}\right)= \\
= & \frac{1}{2} \sum(-1)^{q+1} q\left\{2 ( \operatorname { l o g } 2 ) \left[\operatorname{dim}\left(A_{+}^{q} \cap A_{-}^{q} \oplus R_{+}^{q-1} \cap R_{-}^{q-1}\right)\right.\right. \\
& \left.+\operatorname{dim}\left(R_{+}^{q} \cap R_{-}^{q} \oplus A_{+}^{q-1} \cap A_{-}^{q-1}\right)\right] \\
& +(\log 2)\left[\operatorname{dim}\left(A_{+}^{q} \cap R_{-}^{q} \oplus R_{+}^{q-1} \cap A_{-}^{q-1}\right)\right. \\
& \left.+\operatorname{dim}\left(R_{+}^{q} \cap A_{-}^{q} \oplus A_{+}^{q-1} \cap R_{-}^{q-1}\right)\right] \\
& \left.+\log \operatorname{det}\left(\operatorname{Id}-S_{+}^{q, r} S_{-}^{q, r}\right)+\log \operatorname{det}\left(\operatorname{Id}-S_{+}^{q-1, r} S_{-}^{q-1, r}\right)\right\} \\
= & \sum(-1)^{q}(\log 2)\left[\operatorname{dim}\left(A_{+}^{q} \cap A_{-}^{q}\right)+\operatorname{dim}\left(R_{+}^{q} \cap R_{-}^{q}\right)\right. \\
& \left.+\frac{1}{2} \operatorname{dim}\left(A_{+}^{q} \cap R_{-}^{q}\right)+\frac{1}{2} \operatorname{dim}\left(R_{+}^{q} \cap A_{-}^{q}\right)\right]+\frac{1}{2} \log \operatorname{det}\left(\operatorname{Id}-S_{+}^{q, r} S_{-}^{q, r}\right) .
\end{aligned}
$$

The linear map in the last term may be rewritten

$$
\mathrm{Id}-S_{+}^{q, r} S_{-}^{q, r}=2\left(\operatorname{proj} A_{+}^{q, r} \operatorname{proj} R_{-}^{q, r}+\operatorname{proj} R_{+}^{q, r} \operatorname{proj} A_{-}^{q, r}\right) .
$$

As $A_{+}^{q, r}, R_{-}^{q, r}$ and $R_{+}^{q, r}, A_{-}^{q, r}$ form two pairs of orthocomplements and all have the same dimension, it follows

$$
\frac{1}{2} \log \operatorname{det}\left(\operatorname{Id}-S_{+}^{q, r} S_{-}^{q, r}\right)=\frac{\log 2}{2}\left(\operatorname{dim} A_{+}^{q, r}+\operatorname{dim} R_{-}^{q, r}\right)+\log \operatorname{det}\left(A_{+}^{q, r} \rightarrow R_{-}^{q, r}\right),
$$


where the last operator is orthogonal projection. The formula for the analytic torsion is therefore

$$
\begin{aligned}
\log T\left(M, \mu^{i}\right)= & \log ^{b} T\left(M_{+}, g_{0}\right)+\log ^{b} T\left(M_{-}, g_{0}\right) \\
& +\sum(-1)^{q}\left\{( \operatorname { l o g } 2 ) \left[\operatorname{dim}\left(A_{+}^{q} \cap A_{-}^{q}\right)+\operatorname{dim}\left(R_{+}^{q} \cap R_{-}^{q}\right)\right.\right. \\
& \left.+\frac{1}{2} \operatorname{dim}\left(A_{+}^{q} \cap R_{-}^{q}\right)+\frac{1}{2} \operatorname{dim}\left(R_{+}^{q} \cap A_{-}^{q}\right)\right] \\
& \left.+\log \operatorname{det}\left(A_{+}^{q, r} \rightarrow R_{-}^{q, r}\right)+\frac{\log 2}{2}\left(\operatorname{dim} A_{+}^{q, r}+\operatorname{dim} R_{-}^{q, r}\right)\right\} .
\end{aligned}
$$

Since $\operatorname{dim}\left(A_{+}^{q} \cap A_{-}^{q}\right)+\operatorname{dim}\left(R_{+}^{q} \cap R_{-}^{q}\right)+\operatorname{dim}\left(A_{+}^{q} \cap R_{-}^{q}\right)+\operatorname{dim}\left(R_{+}^{q} \cap A_{-}^{q}\right)+$ $\operatorname{dim} A_{+}^{q, r}+\operatorname{dim} R_{-}^{q, r}=\operatorname{dim} H^{q}(H)$, the formula above becomes

$$
\begin{aligned}
& \log T\left(M, \mu^{i}\right)=\log { }^{b} T\left(M_{+}, g_{0}\right)+\log ^{b} T\left(M_{-}, g_{0}\right) \\
& +\sum(-1)^{q}\left\{\frac{\log 2}{2}\left[\operatorname{dim}\left(A_{+}^{q} \cap A_{-}^{q}\right)+\operatorname{dim}\left(R_{+}^{q} \cap R_{-}^{q}\right)\right]\right. \\
& \left.+\log \operatorname{det}\left(A_{+}^{q, r} \rightarrow R_{-}^{q, r}\right)\right\}+\frac{\log 2}{2} \chi_{E}(H) .
\end{aligned}
$$

\section{Surgery formula for R-torsion.}

Let

$$
0 \rightarrow K_{1} \stackrel{k_{1}}{\longrightarrow} K_{2} \stackrel{k_{2}}{\longrightarrow} K_{3} \rightarrow 0
$$

be an exact sequence of complexes with inner product, such that the induced volumes are compatible. This means that, if $a_{1}^{q}$ is an orthonormal basis for $K_{1}^{q}, a_{2}^{q}$ an orthonormal basis in $K_{2}^{q}$ and $a_{3}^{q}$ an independent set in $K_{2}^{q}$ mapping to an orthonormal basis of $K_{3}^{q}$, then

$$
\left[k_{1}^{q}\left(a_{1}^{q}\right), a_{3}^{q} \mid a_{2}^{q}\right]=1 .
$$

Let elements of cohomology $\mu_{i}^{q}$ be given in $H^{q}\left(K_{i}\right)$. With these chosen volume elements, the long exact sequence in cohomology

$$
\rightarrow H^{q-1}\left(K_{3}\right) \rightarrow H^{q}\left(K_{1}\right) \stackrel{k_{1}^{q}}{\longrightarrow} H^{q}\left(K_{2}\right) \stackrel{k_{2}^{q}}{\longrightarrow} H^{q}\left(K_{3}\right) \rightarrow
$$

is an acyclic complex $\mathcal{H}$ with volumes. Then there is a formula (see [14])

$$
\tau\left(K_{2}\right)=\tau\left(K_{1}\right) \tau\left(K_{3}\right) \tau(\mathcal{H}) .
$$


The behaviour of R-torsion under surgery follows readily from this formula.

Suppose that $M$ is odd dimensional and is given a simplicial decomposition such that $H$, and therefore $M_{+}$and $M_{-}$, are subcomplexes. Apply (37) to the exact sequence in simplicial cohomology

$$
0 \rightarrow C_{\mathrm{rel}}^{*}\left(M_{ \pm}\right) \stackrel{i}{\rightarrow} C^{*}(M) \stackrel{p}{\rightarrow} C_{\mathrm{abs}}^{*}\left(M_{\mp}\right) \rightarrow 0 .
$$

Here, relative cochains on $M_{ \pm}$are those which vanish at the boundary; absolute cochains are unrestricted at the boundary. With the usual inner products on these spaces (all delta functions on cells orthonormal) this short exact sequence is compatible on induced volumes, so (37) applies. This equation is, in logarithmic form,

$$
\log \tau(M)=\log \tau\left(M_{+}\right)+\log \tau\left(M_{-}\right)+\log \tau(\mathcal{H}),
$$

where it is understood that $\log \tau(\mathcal{H})$ is measured with respect to the same choices of volumes that were used in to compute the other torsions. To use this result, one must calculate $\tau(\mathcal{H})$, where $\mathcal{H}$ is the long exact sequence

$$
\rightarrow H_{\mathrm{abs}}^{q-1}\left(M_{\mp}\right) \stackrel{c^{q-1}}{\longrightarrow} H_{\mathrm{rel}}^{q}\left(M_{ \pm}\right) \stackrel{i^{q}}{\longrightarrow} H^{q}(M) \stackrel{p^{q}}{\longrightarrow} H_{\mathrm{abs}}^{q}\left(M_{\mp}\right) \stackrel{c^{q}}{\longrightarrow}
$$

To compare $\tau(M)$ to the analytic torsion $T\left(M, \mu^{i}\right)$, take volumes in cohomology given by an orthonormal basis $\mu^{i}$ of surgery Hodge forms defined in equation (22), and volumes $\nu_{ \pm}^{i}$ for the relative or absolute cohomology of $M_{ \pm}$given by b-Hodge theory. Split these spaces into subspaces corresponding to the images of the above maps and their orthogonal complements:

$$
\begin{aligned}
H_{\mathrm{rel}}^{q}(M \pm) \equiv\left[\left(R_{ \pm}^{q-1} \ominus R_{\mp}^{q-1}\right)\right] \oplus\left[H_{b-\mathrm{Ho}}^{q}\left(M_{ \pm}\right) \oplus\left(R_{+}^{q-1} \cap R_{-}^{q-1}\right)\right] \\
H^{q}(M) \equiv\left[H_{b-\mathrm{Ho}}^{q}\left(M_{ \pm}\right) \oplus \sqrt{2}\left(R_{+}^{q-1} \cap R_{-}^{q-1}\right)\right] \oplus \\
\\
{\left[H_{b-\mathrm{Ho}}^{q}\left(M_{\mp}\right) \oplus \sqrt{2}\left(A_{+}^{q} \cap A_{-}^{q}\right)\right] } \\
H_{\mathrm{abs}}^{q}(M \mp) \equiv\left[H_{b-\mathrm{Ho}}^{q}\left(M_{\mp}\right) \oplus\left(A_{+}^{q} \cap A_{-}^{q}\right)\right] \oplus\left[\left(A_{\mp}^{q} \ominus A_{ \pm}^{q}\right)\right] .
\end{aligned}
$$

Factors of $\sqrt{2}$ indicate that the inner product to be taken is $\sqrt{2}$ times the standard one. They come from the factors of two in (22); with these inner products this decomposition is isometric. Referring to (18), the torsion is given by

$$
\begin{aligned}
\log \tau(\mathcal{H})= & \sum(-1)^{q} \log \operatorname{det} i^{q}\left\lceil H_{b-\mathrm{Ho}}^{q}\left(M_{ \pm}\right) \oplus R_{+}^{q-1} \cap R_{-}^{q-1}\right. \\
& -\log \operatorname{det} p^{q}\left\lceil H_{b-\mathrm{Ho}}^{q}\left(M_{\mp}\right) \oplus \sqrt{2} A_{+}^{q} \cap A_{-}^{q} \oplus \sqrt{2} R_{+}^{q-1} \cap R_{-}^{q-1}\right. \\
& +\log \operatorname{det} c^{q}\left\lceil\left(A_{\mp}^{q} \ominus A_{ \pm}^{q}\right) .\right.
\end{aligned}
$$


Recall the explicit definitions of the maps $i^{q}, p^{q}$ and $c^{q}$. The map $i$ takes a relative form on $M_{ \pm}$, cuts off near the boundary to get a form on $M$, and then projects into Hodge cohomology. On the space $H_{b-\mathrm{Ho}}^{q}\left(M_{ \pm}\right)$this map is the identity and on $R_{+}^{q-1} \cap R_{-}^{q-1}$ this map is multiplication by $1 / 2$, which means it has $\log$ determinant $\log (1 / \sqrt{2})\left(\operatorname{dim} R_{+}^{q-1} \cap R_{-}^{q-1}\right)$ on this factor.

The map $p$ is restriction to $M_{ \pm}$; this is the identity on $H_{b-\mathrm{Ho}}^{q}\left(M_{\mp}\right)$ and on $A_{+}^{q} \cap A_{-}^{q}$, which means it has $\log$ determinant $\log (1 / \sqrt{2})\left(\operatorname{dim} A_{+}^{q} \cap A_{-}^{q}\right)$ on this factor. To find the image of the connecting homomorphism $c$ one takes $\beta \in A_{\mp}^{q} \ominus A_{ \pm}^{q}$, extends into $M$, applies $d$ and regards the result as a form in $H_{\text {rel }}^{q}(M \pm)$; it is projection from $A_{\mp}^{q} \ominus A_{ \pm}^{q}$ to $R_{ \pm}^{q} \ominus R_{\mp}^{q}$. Thus,

$$
\begin{gathered}
\log \tau(\mathcal{H})=\sum(-1)^{q}\left\{\left(-\frac{\log 2}{2}\right) \operatorname{dim}\left(R_{+}^{q-1} \cap R_{-}^{q-1}\right)+\frac{\log 2}{2} \operatorname{dim}\left(A_{+}^{q} \cap A_{-}^{q}\right)\right. \\
\left.+\log \operatorname{det}\left(A_{\mp}^{q} \ominus A_{ \pm}^{q} \rightarrow R_{ \pm}^{q} \ominus R_{\mp}^{q}\right)\right\} \\
=\sum(-1)^{q}\left\{\frac{\log 2}{2}\left(\operatorname{dim}\left(A_{+}^{q} \cap A_{-}^{q}\right)+\operatorname{dim}\left(R_{+}^{q} \cap R_{-}^{q}\right)\right)+\log \operatorname{det}\left(A_{+}^{q, r} \rightarrow R_{-}^{q, r}\right)\right\}
\end{gathered}
$$

Again the arrow denotes orthogonal projection. Subtracting this from (35) yields Theorem 1 .

\section{Combinatorial formulae for analytic and b-analytic torsion.}

In this final section the Cheeger-Müller theorem and Theorem 2 are readily obtained as corollaries of Theorem 1 .

Proposition 7 (Cheeger-Müller Theorem). If $E$ is a flat unitary bundle over a closed manifold $M$ then $T(M ; E)=\tau(M ; E)$.

Proof. If $M$ is even dimensional then both torsions are equal to 1 . So assume that $M$ is odd dimensional. Then the result follows by applying Theorem 1 to a series of Morse surgeries that transform two copies of any manifold $M$ to the sphere $S^{n}$, as Cheeger does in [5].

Let $f$ be a nonnegative Morse function on $M$ with distinct critical values. Define $F$ on $M \times[0,1]_{u}$ by $F(p, u)=4 u(1-u) f(p)$. Then the critical points of $F$ are $(p, 1 / 2)$ where $p$ is a critical point of $f$. Since $F(p, 1 / 2)=f(p)$ at critical points, $F$ has distinct critical values. Let $a=\max f$. For $\delta$ small 
enough, $F^{-1}(a-\delta)$ is an $n$-sphere, and $F^{-1}[0, a-\delta]$ is a manifold with boundary $2 M \cup S^{n}$ such that $F$ attains a minimum on $2 M$, a maximum on $S^{n}$ and has nonvanishing differential at the boundary. Analytic and Rtorsion are both unchanged by a change of orientation and are multiplicative under disjoint union, so it suffices to prove the Proposition for $2 M$. The level sets of $F$ define a family of manifolds that are diffeomorphic except when a critical point of $F$ is crossed, in which case a cell is attached. Thus, from this one gets a finite collection of manifolds $M_{0}=S^{n}, M_{1}, \ldots M_{N}=2 M$, such that $M_{i+1}$ is obtained from $M_{i}$ by removing a $S^{k} \times D^{n-k}$ and gluing in a $D^{k+1} \times S^{n-k-1}$ along the (common) boundary. For any flat unitary bundle $E$ over $M$, the lift of $E$ to $M \times[0,1]$ restricted to $F^{-1}(b)$ is a flat unitary bundle $E_{b}$ over $f^{-1}(b)$. If there are no critical values of $F$ in $\left[b_{1}, b_{2}\right]$ then the bundles $E_{b_{1}}$ and $E_{b_{2}}$ are isomorphic flat bundles, so one can write $E_{i}$ for the flat bundle over $M_{i}$. Since the surgery occurs in an arbitrarily small coordinate neighbourhood of a point in $M \times[0,1]$, the lift of the bundle $E$ is trivial there. Therefore $E_{i}$ is trivial on $S^{k} \times D^{n-k}$ and the bundle $E_{i+1}$ is obtained from $E_{i}$ by gluing in a trivial bundle over $D^{k+1} \times S^{k-1}$.

The equality of $T$ and $\tau$ for spheres, and therefore products of spheres, is known, due to explicit calculations by Ray in [17]. The proof proceeds by showing inductively that $T\left(M_{i+1} ; E_{i+1}\right)=\tau\left(M_{i+1} ; E_{i+1}\right)$ assuming that $T\left(M_{i} ; E_{i}\right)=\tau\left(M_{i} ; E_{i}\right)$. Note that all these surgeries involve a separating hypersurface, although sometimes it is disconnected.

Now apply Theorem 1. First, with $M=S^{k} \times S^{n-k}$, and $M_{ \pm}=S^{k} \times$ $D^{n-k}$, with trivial bundle $F$,

$$
\log \frac{{ }^{b} T\left(S^{k} \times D^{n-k} ; F\right)}{\tau\left(S^{k} \times D^{n-k} ; F\right)}=-\frac{1}{4} \chi\left(S^{k} \times S^{n-k}\right) \operatorname{dim} F=0,
$$

since $n$ is odd. Applying it to $M_{i}, M_{i,+}=M_{i} \backslash\left(S^{k} \times D^{n-k}\right), M_{i,-}=$ $S^{k} \times D^{n-k}$, and assuming the result for $\left(M_{i} ; E_{i}\right)$ yields

$$
\log \frac{{ }^{b} T\left(M_{i,+} ; E_{i}\right)}{\tau\left(M_{i,+} ; E_{i}\right)}=0 .
$$

Finally, applying it to $M_{i+1}, M_{i+1,+}=M_{i+1} \backslash\left(D^{k+1} \times S^{n-k-1}\right), M_{i+1,-}=$ $D^{k+1} \times S^{n-k-1}$ yields

$$
\log \frac{T\left(M_{i+1} ; E_{i+1}\right)}{\tau\left(M_{i+1} ; E_{i+1}\right)}=\log \frac{{ }^{b} T\left(M_{i+1,+} ; E_{i+1}\right)}{\tau\left(M_{i+1,+} ; E_{i+1}\right)} .
$$

Since $\left(M_{i,+} ; E_{i}\right)=\left(M_{i+1,+} ; E_{i+1}\right)$, the Cheeger-Müller Theorem follows. 
Proof of Theorem 2. Using the metric independence (24) of b-analytic torsion, one can assume that $N$ is an exact product near the boundary. Then by doubling $N$ and applying Theorem 1, (2) follows.

Example 8. The interval $[0,1]$. Consider the trivial $\mathbb{C}$-bundle $E$ over the interval $[0,1]_{x}$, with b-metric $d x^{2} /\left(x^{2}(1-x)^{2}\right)$. Since this makes $[0,1]$ isometric to $\mathbb{R}$ and the Laplacian on $[0,1]$ equivalent to the usual Laplacian on $\mathbb{R}$, all b-Traces vanish and so $\log ^{b} T([0,1] ; E)=0$, whence ${ }^{b} T([0,1] ; E)=1$. This can be compared to the R-torsion relative to an orthonormal basis of b-Hodge cohomology (relative or absolute). The $L^{2}$ cohomology of $\mathbb{R}$ is $\{0\}$, and the absolute cohomology consists of constant zero-forms. An orthonormal basis of $H_{b \text {-abs,Ho }}^{*}([0,1])$ is therefore $\{1 / \sqrt{2}\}$ since $1 / \sqrt{2}$ has boundary data $(1 / \sqrt{2}, 1 / \sqrt{2})$ which has unit length. The R-torsion can be calculated by applying (18) to the cochain complex given by the cell decomposition $e=[0,1], P_{0}=\{0\}, P_{1}=\{1\}$. With $\mu_{0}=\left\{1 / \sqrt{2} \delta_{P_{0}}+1 / \sqrt{2} \delta_{P_{1}}\right\}$, the $\mathrm{R}$-torsion is equal to

$$
\tau([0,1])=\left|\operatorname{det} d_{\perp}^{0}\right|=\left|d^{0}\left(\frac{1}{\sqrt{2}} \delta_{P_{0}}-\frac{1}{\sqrt{2}} \delta_{P_{1}}\right)\right|=\left|\sqrt{2} \delta_{e}\right|=\sqrt{2} .
$$

One can also calculate analytic torsion of the Laplacian on $[0,1]$ with respect to absolute boundary conditions on $[0,1]$. The form $a(x)+b(x) d x$ satisfies absolute boundary conditions on $[0,1]$ if $\partial_{x} a(0)=\partial_{x} a(1)=b(0)=$ $b(1)=0$. The form $1 / \sqrt{2}$ is an orthonormal basis of absolute cohomology if the length of $[0,1]$ is two. The nonzero spectrum of the Laplacian on 1-forms is then $\left(\frac{n \pi}{2}\right)^{2}, n>0$, so

$$
\zeta_{1}(s)=\left(\frac{\pi}{2}\right)^{-2 s} \zeta(2 s)
$$

with $\zeta$ the Riemann zeta function. Thus

$$
\begin{aligned}
\log T=-\frac{1}{2} \zeta_{1}^{\prime}(0) & =-\frac{1}{2}\left[-2 \log \frac{\pi}{2} \zeta(0)+2 \zeta^{\prime}(0)\right] \\
& =-\frac{1}{2}\left[\log \frac{\pi}{2}-\log 2 \pi\right] \\
& =-\frac{1}{2} \log \frac{1}{4} \\
& =\log 2,
\end{aligned}
$$

so $T=2$. Thus the formulae ${ }^{b} T(N)=\tau(N) 2^{-1 / 4 \chi(\partial N)}$ and $T(N)=$ $\tau(N) 2^{1 / 4 \chi(\partial N)}$ for absolute boundary conditions are verified in this case. 


\section{Appendix: Long time behaviour of the b-trace of the heat kernel.}

Let $N$ be a manifold with boundary $H$, with exact b-metric $g$, let $\Delta_{N}$ be a generalized Laplacian corresponding to $g$, and let $\Delta_{H}$ denote the Laplacian restricted to the boundary. In [11] it was shown that $b-\operatorname{Tr} e^{-t \Delta_{N}}$ has an expansion as $t \rightarrow \infty$

$$
b-\operatorname{Tr} e^{-t \Delta_{N}}=\sum_{j=0}^{k} a_{j} t^{-j / 2}+O\left(t^{-(k+1) / 2}\right)
$$

for any $k$. If null $\Delta_{H}=\{0\}$, then $a_{0}=\operatorname{dim} L^{2}$ null $\Delta_{N}$, but in general this is not the case. This result is at first surprising since the heat kernel itself equals $\operatorname{proj} L^{2}$ null $\Delta_{N}+O\left(t^{-1 / 2}\right)$. The extra term arises from the non-uniformity of the b-regularization of certain terms in the heat kernel as $t \rightarrow \infty$. This was observed in [11], but the value of $a_{0}$ was calculated incorrectly. In this appendix the correct value is computed.

It was shown in section 8 of Part II that the surgery heat kernel, restricted to the diagonal, is smooth on the logarithmic heat space $\Delta_{\mathrm{LHs}}$, except, possibly, at the face $B_{\mathrm{t} \infty}$. Restricting to the face $B_{0}=\bar{M}_{\log }$ shows that the b-heat kernel $e^{-t \Delta_{0}}$ on the diagonal is smooth on

$$
\left[\bar{M}_{\log } \times[0, \infty]_{\tau} ;\{\operatorname{ilg} x=1 / \tau=0\}\right] .
$$

In fact, this is true for any manifold with boundary $N$.

The b-Trace of the heat kernel on $N$ is the result of a b-pushforward from this space to $[0, \infty]_{\tau}$. By Lemma 9 , Part II, the coefficient $a_{0}$ is the sum of a b-integral over each of the two boundary hypersurfaces at $\tau=\infty$, namely $N_{\log } \times\{\tau=\infty\}$ and the blowup of $\{$ ilg $x=1 / \tau=0\}$, which will be denoted $K$. The former b-integral is absolutely convergent and yields the 'expected' value $\operatorname{dim} L^{2}$ null $\Delta_{N}$. The latter yields the 'extra' term. The value of the heat kernel on $K$ can be found by matching with the value on the face $B_{2}^{\infty}$, since in $\Delta_{\mathrm{LHs}}, K$ is the intersection of $M_{\mathrm{log}} \equiv B_{0}$ and $B_{2}^{\infty}$. By (7), this is (ias $\epsilon) e^{-T \operatorname{RN}(\Delta)}(s, s)$ and the intersection with $K$ occurs at $s= \pm 1, T=0$. Referring to Part II, section 5, the heat kernel $e^{-T \operatorname{RN}(\Delta)}$ has the form

$$
e^{-T \mathrm{RN}(\Delta)}=\frac{1}{\sqrt{4 \pi T}}\left(e^{-\left|s-s^{\prime}\right|^{2} / 4 T} \operatorname{Id}+e^{-\left| \pm 2-s-s^{\prime}\right|^{2} / 4 T} S_{ \pm}\right)+O\left(e^{-c / T}\right) .
$$

Write $\xi=\operatorname{ilg} x, \rho=1 / \tau$, and $\sigma=\xi / \rho$; then $\sigma$ is a coordinate along the quarter circles fibreing $K$. In terms of $s$ and $T, \sigma=\sqrt{T} /(1-s)$. Writing 
$d h$ for the metric on $H$, the canonical density $d g d t / t$ at $K$ is

$$
\frac{d \xi}{\xi^{2}} d h \frac{d t}{t}=\left(\tau \frac{d \sigma}{\sigma^{2}} d h \frac{d t}{t}\right)
$$

Thus, if $S_{J K}$ is the scattering matrix for $N$, the contribution from $K$ is a regularization of the divergent integral

$$
\left(\int_{0}^{\infty} \frac{1}{\sqrt{4 \pi}} \int_{H}\left(\phi_{J} \phi_{J}+e^{-1 / \sigma^{2}} S_{J K}(0) \phi_{J} \phi_{K}\right) \frac{d \sigma}{\sigma^{2}}\right) \frac{d t}{t} .
$$

By Lemma 9 of Part II, the first, divergent term yields the same contribution as the term

$$
\left(b \int_{N} \frac{1}{\sqrt{4 \pi}} \frac{1}{\tau} \phi_{J} \phi_{J} d g\right) \frac{d t}{t}
$$

cut off away from the boundary, lifted to the blown up space and restricted to $K$. Since $1 / \tau$ is constant on the fibres of the pushforward map, this is equal to

$$
\frac{1}{\tau}\left(\int_{N} \frac{1}{\sqrt{4 \pi}} \phi_{J} \phi_{J} d g\right) \frac{d t}{t}
$$

and is therefore $O(1 / \tau)$, giving zero contribution to $a_{0}$. The other term is absolutely integrable and yields

$$
\begin{aligned}
\left(\frac{1}{\sqrt{4 \pi}} \operatorname{tr} S(0) \int_{0}^{\infty} e^{-1 / \sigma^{2}} \frac{d \sigma}{\sigma^{2}}\right) \frac{d t}{t}=\left(\frac{1}{4}\right. & \operatorname{tr} S(0)) \frac{d t}{t} \\
& =\left(\frac{1}{4}\left(\operatorname{dim} \Lambda^{D}-\operatorname{dim} \Lambda^{N}\right)\right) \frac{d t}{t}
\end{aligned}
$$

by changing variable to $1 / \sigma$ and using (4). Cancelling the formal density factor $d t / t$ on $\mathbb{R}$ yields

$$
\begin{aligned}
a_{0} & =\operatorname{dim} L^{2} \text { null } \Delta_{N}+\frac{1}{4}\left(\operatorname{dim} \Lambda^{D}-\operatorname{dim} \Lambda^{N}\right) \\
& =\operatorname{dim} L^{2} \text { null } \Delta_{N}+\frac{1}{2} \operatorname{dim} \Lambda^{D}-\frac{1}{4} \operatorname{dim} \text { null } \Delta_{H} .
\end{aligned}
$$

This differs from the value claimed in [11] by $-1 / 4 \operatorname{dim}$ null $\Delta_{H}$. Notice that, in the APS index theorem to which this result is applied in [11], this term occurs in both $b$ - Tr $e^{-t \Im^{+} \mathfrak{g}^{-}}$and $b-\operatorname{Tr} e^{-t \mathfrak{\Im}^{-\Im^{+}}}$, and therefore cancels. In the present situation, however, it does not cancel. 
In the formula for b-analytic torsion of $\bar{M}$ there is a term proportional to Euler's constant $\gamma$, analogous to the term in (10). By (39) this term is given by

$$
\begin{aligned}
\frac{\gamma}{2} \sum(-1)^{q} q\left(\operatorname{dim} L^{2}\right. & \operatorname{null} \Delta_{q, \bar{M}}+\frac{1}{2}\left(\operatorname{dim} \Lambda_{q,+}^{D}+\Lambda_{q,-}^{D}\right) \\
& \left.-\frac{1}{4}\left(\operatorname{dim} \operatorname{null} \Delta_{q, M_{+}} \uparrow H+\operatorname{dim} \operatorname{null} \Delta_{q, M_{-}} \uparrow H\right)\right) .
\end{aligned}
$$

As $\Delta_{q, M_{ \pm}}\left\lceil H=\Delta_{q, H} \oplus \Delta_{q-1, H}\right.$, this is

$$
\begin{aligned}
= & \frac{\gamma}{2} \sum(-1)^{q} q\left(\operatorname{dim} L^{2} \operatorname{null} \Delta_{q, \bar{M}}+\frac{1}{2}\left(\operatorname{dim} A_{+}^{q}+\operatorname{dim} R_{+}^{q-1}\right.\right. \\
& \left.\left.+\operatorname{dim} A_{-}^{q}+\operatorname{dim} R_{-}^{q-1}\right)-\frac{1}{2}\left(\operatorname{dim} \operatorname{null} \Delta_{q, H}+\operatorname{dim} \operatorname{null} \Delta_{q-1, H}\right)\right) \\
= & \frac{\gamma}{2}\left(\sum(-1)^{q} q \operatorname{dim} L^{2} \operatorname{null} \Delta_{q, \bar{M}}\right. \\
& +\sum(-1)^{q} \frac{q}{2}\left(\left(\operatorname{dim} \operatorname{null} \Delta_{q, H}-\operatorname{dim} R_{+}^{q}\right)+\operatorname{dim} R_{+}^{q-1}+\operatorname{dim} A_{-}^{q}\right. \\
& \left.\left.+\left(\operatorname{dim} \operatorname{null} \Delta_{q-1, H}-A_{-}^{q-1}\right)-\frac{1}{2}\left(\operatorname{dim} \operatorname{null} \Delta_{q, H}+\operatorname{dim} \operatorname{null} \Delta_{q-1, H}\right)\right)\right) \\
= & \frac{\gamma}{2}\left(\sum(-1)^{q} q \operatorname{dim} L^{2} \operatorname{null} \Delta_{q, \bar{M}}\right. \\
& \left.+\sum(-1)^{q} \frac{q}{2}\left(\operatorname{dim} R_{+}^{q-1}-\operatorname{dim} R_{+}^{q}+\operatorname{dim} A_{-}^{q}-\operatorname{dim} A_{-}^{q-1}\right)\right) \\
= & \frac{\gamma}{2}\left(\sum(-1)^{q} q \operatorname{dim} L^{2} \operatorname{null} \Delta_{q, \bar{M}}+\sum(-1)^{q} \frac{1}{2}\left(\chi_{\text {abs }}\left(M_{-}\right)+\chi_{\text {rel }}\left(M_{+}\right)\right)\right)
\end{aligned}
$$

since $\operatorname{dim} H_{\mathrm{abs}}^{q}\left(M_{-}\right)=\operatorname{dim} A_{-}^{q}+\operatorname{dim} L^{2}$ null $\Delta_{q, M_{-}}$and $\operatorname{dim} H_{\mathrm{rel}}^{q}\left(M_{+}\right)=$ $\operatorname{dim} R_{+}^{q-1}+\operatorname{dim} L^{2}$ null $\Delta_{q, M_{+}}$and $\chi_{L^{2}}\left(M_{ \pm}\right)=0$ since $M$ is odd dimensional. By exactness of the sequence (38),

$$
\chi_{\text {abs }}\left(M_{-}\right)-\chi(M)+\chi_{\text {rel }}\left(M_{+}\right)=0 .
$$

As $\chi(M)=0$, this shows $\chi_{\text {abs }}\left(M_{-}\right)+\chi_{\text {rel }}\left(M_{+}\right)=0$. Hence the term calculated above is just

$$
\frac{\gamma}{2} \sum(-1)^{q} q \operatorname{dim} L^{2} \text { null } \Delta_{q, \bar{M}}
$$




\section{References.}

[1] J.-M. Bismut and W. Zhang, An extension of the Cheeger-Müller theorem, Astérisque 205, Paris, 1992.

[2] U. Bunke, A glueing formula for the eta invariant, J. Diff. Geom. 41(1995),397448.

[3] D. Burghelea, L. Friedlander, T. Kappeler, Asymptotic expansion of the Witten deformation of the analytic torsion, J. Funct. Anal. 137 (1996), 320-363.

[4] S. E. Cappell, R. Lee and E. Y. Miller, Self-adjoint operators and manifold decomposition Part I: Low eigenmodes and stretching, Comm. Pure Appl. Math. 49(1996), 825-866.

[5] J. Cheeger, Analytic torsion and the heat equation Annals of Math. 109 (1979), 259-333.

[6] A. Hassell, R. R. Mazzeo and R. B. Melrose, Analytic surgery and the accumulation of eigenvalues, Comm. in Anal. and Geom. 3 (1995), No. 1, 115-222.

[7] S. Klimek and K. Wojciechowski. Adiabatic cobordism theorems for analytic torsion and $\eta$-invariant, J. Funct. Anal. 136(1996), 269-293.

[8] W. Lück. Analytic and topological torsion for manifolds with boundary and symmetry, Jour. Diff. Geom. 37 (1993), 263-322.

[9] R. R. Mazzeo and R. B. Melrose, Analytic surgery and the eta invariant, Geom. Funct. Anal. 5 (1995), No. 1, 14-75.

[10] P. McDonald, The Laplacian on spaces with conic singularities, Thesis, MIT (1990).

[11] R. B. Melrose, The Atiyah-Patodi-Singer index theorem, A. K. Peters, 1993.

[12] Calculus of conormal distributions on manifolds with corners, International Mathematics Research Notices (1992), No. 3, 51-61.

[13] , Pseudodifferential Operators, Corners and Singular Limits, Proceedings of the International Congress of Mathematicians (Kyoto, 1990), Math. Soc. Japan (1991), 217-234.

[14] J. W. Milnor, Whitehead torsion, Bull. Amer. Math. Soc. 72 (1966), 358-426.

[15] W. Müller, Analytic torsion and R-torsion of Riemannian manifolds, Adv. in Math. 28 (1978), 233-305. 
[16] W. Müller, Analytic torsion and R-torsion for unimodular representations, Jour. Amer. Math. Soc. 6 (1993), 721-753.

[17] D. B. Ray, R-torsion and the Laplacian on Lens Spaces, Adv. in Math. 4 (1970), 109-126.

[18] D. B. Ray and I. M. Singer, R-torsion and the Laplacian on Riemannian Manifolds, Adv. in Math. 7 (1971), 145-210.

[19] D. B. Ray and I. M. Singer, Analytic Torsion, in Partial Differential Equations, Proc. Symp. Pure Math. XXIII, Amer. Math. Soc. (1971), pp. 167-181.

[20] S. M. Vishik, Analytic torsion of boundary value problems, Sov. Math. Dokl. 36, No. 1 (1988), 174-179.

[21] S. M. Vishik, Generalized Ray-Singer Conjecture I, Comm. Math. Phys. 167(1995), 1-102.

ReCEIVED February 26, 1996.

Centre for Mathematics and Its Applications

A.N.U.

Canberra ACT 0200

Australia

E-mail address: HASSELLA@MATHS.ANU.EDU.AU 